\title{
Asymptotic Stochastic Transformations for Nonlinear Quantum Dynamical Systems
}

\author{
John Gough
}

\begin{abstract}
The Ito and Stratonovich approaches are carried over to quantum stochastic systems. Here the white noise representation is shown to be the most appropriate as here the two approaches appear as Wick and Weyl orderings, respectively. This introduces for the first time the Stratonovich form for SDEs driven by Poisson processes or quantum SDEs including the conservation process. The relation of the nonlinear Heisenberg ODES to asymptotic quantum SDEs is established extending previous work on linear (Schrodinger) equations. This is shown to generalize the classical integral transformations between the various forms of stochastic calculi and to extend the Khasminskii theorem to the quantum setting.
\end{abstract}

\section{Introduction}

The stochastic asymptotic analysis of dynamical systems has its origins in Einstein's theory of Brownian motion, however, it extends to very general classes of systems, both classical and quantum.

In the classical case, one considers typically a dynamical variable $x_{t}=x_{t}(\lambda)$ determined from an ODE $\frac{d}{d t} x_{t}(\lambda)=$ $\lambda F\left(x_{t}(\lambda), M_{t}\right), x_{0}(\lambda)=x_{0}$, where $\lambda>0$ is a coupling parameter, $M_{t}$ is an external (stochastic) input and $F$ is some function (usually Lipschitz with bounded first partial derivatives). Supposing that $\frac{1}{T} \int_{t}^{T+t} d s F\left(x, M_{s}\right)$ converges as $T \rightarrow \infty$ in probability to $F_{o}(x)$ uniformly in $t$ for arbitrary $x$, then under suitable conditions [1] the averaging principle states that $x_{t / \lambda}(\lambda)$ converges in probability to $\bar{x}_{t}$, the solution of the averaged ODE $\frac{d}{d t} \bar{x}_{t}=F_{o}\left(\bar{x}_{t}\right) ; \bar{x}_{0}=x_{0}$. One may think of $x_{t / \lambda}(\lambda)$ as a perturbation away from the averaged solution. If $F_{o}=0$, the averaging principle states that $x_{t / \lambda}(\lambda)$ will not vary significantly from $x_{0}$ on time scales $[0, T / \lambda][2]$. This lead Stratonovich in 1961 to suggest that on time scales $\left[0, T / \lambda^{2}\right]$ stochastic fluctuations accumulate appreciably; this idea was substantiated by Khasminskii [3] who proved that $x_{t / \lambda^{2}}(\lambda)$ converges weakly to a Markov diffusion. In this context, the interpretation in terms of a white noise Langevin equation, originally given by Wong and Zakai [4], has been revealing. If $\xi(t)$ are regular stochastic processes with mean zero and correlation $\mathbb{E}[\xi(t) \xi(s)]=\frac{1}{\lambda^{2}} c\left(\frac{t-s}{\lambda^{2}}\right)$, so that the $\lambda \rightarrow 0$ limit leads to a white noise process, then the dynamical - equations of motion can converge to an asymptotic Langevin equation which retains the pre-limit form provided one uses the Stratonovich version of stochastic calculus [5].

When dealing with quantum systems the scaling employed in the Khasminskii theorem corresponds to the van Hove re-scaling of the dynamical variables [6]. The formulations of most quantum Langevin equations encountered in physics [7] can occasionally be justified as an asymptotic quantum stochastic limit of such re-scaled variables [S]. The germ of the approach to be developed in this paper comes from the work of Accardi, Frigerio and Lu [9] wherein a class of noncommutative Khasminskii theorems are established. The point of view presented here is closer in spirit to that of Wong and Zakai. In particular, quantum white noises [10] play a fundamental role. These are Bose operators (t) satisfying a canonical commutation relation (CCR)

$$
[a(t), a(s))]=\kappa \delta_{+}(t-s)+\kappa^{*} \delta_{-}(t-s),
$$

where $\kappa$ is a complex number and $\delta_{ \pm}(t)$ are special delta functions on the space of regulated functions picking out the future/past values at a time $t$. The form (1.1) arises as the limit form of the CCR for physical fields wherein the righthand side would be typically the causal (Feynman) propagator. The $a^{\sharp}(t)$ have white noise spectra and play a role similar to the input processes studied by Gardiner [11], though with different operational properties and without the effective restriction $\kappa \equiv \frac{1}{2}$. As such, the choice between Ito and Stratonovich versions of stochastic calculus corresponds to the choice between Wick and Weyl ordering, respectively, of the noises with respect to the integrands. Note that the product 
of two Wick ordered expressions will require further re-ordering to attain Wick order again, this leads to extra terms which correspond to the standard shift accounted for by the quantum Ito table [12]. This notion, in fact, dates back to an observation of Hudson and eater [ 131. It should also be mentioned that an alternate description of Stratonovich quantum stochastic calculus, not involving white noise and taking $\kappa=\frac{1}{2}$, has been given independently by Chebotarev [14]. It is worth mentioning that while the stochastic limits described here should preserve canonical structures, especially unitarity, many important physical properties such as detailed balance and the KMS condition are typically lost [15]. Heuristic accounts of these white noise processes have appeared [16, 171 along with an account of their applications to physic [18]. However, only linear systems have been treated up to now. In this paper, the analysis is extended to nonlinear systems which essentially emerge as Heisenberg evolutes corresponding to linear quantum SDEs (i.e. stochastic Schrodinger equations). Complete transformation laws are derived. The classical formulae for Ito-Stratonovich conversion for both Wiener and Poisson driven systems are recovered as special cases.

\section{Classical stochastic differential equations}

In this section several well-known features [19] of SDEs are reviewed. Let $u, \sigma^{(\alpha)} \in C\left({ }^{n} \times \mathbb{R}, \mathbb{R}^{n}\right)$ satisfy appropriate Lipschitz and growth conditions, and let $M_{t}^{(\alpha)}$ be a set of stochastic processes on a probability space $(\Omega, \mathcal{F}, \mathbb{P})$. Let $x_{0} \in L^{2}(\Omega, \mathcal{F}, \mathbb{P})$ be independent of the processes $M_{t}^{(\alpha)}$ for $t>0$. Whenever the $M_{t}^{(\alpha)}$ are nondifferentiable, the formal equation (2.1) below is technically meaningless,

$$
\frac{d X_{t}}{d t}=v\left(X_{t}, t\right)+\sum_{\alpha} \sigma^{(\alpha)}\left(X_{t}, t\right) \frac{d M_{t}^{(\alpha)}}{d t}, \quad X_{0}=x_{0}
$$

Different finite-step numerical schemes used to define an estimate for $X$, in (2.1) lead to inconsistencies in the small time-step limit which do not arise when dealing with ODES. For instance, let $\mathcal{P}$ be an ordered partition of $[0, t]$, say $\left[0=t_{0}<t_{1}<\cdots<t_{N}=t\right]$, and denote $\max \left|t_{j+1}-t_{j}\right|$ by $|\mathcal{P}|$. One constructs an approximate solution $X_{T}^{\mathcal{P}}$ to $(2.1)$ in the form of a random variable $X_{t_{N}}^{\mathcal{P}}$, obtained by the iterative scheme

$$
X_{t_{j+1}}^{\mathcal{P}}=X_{t_{j}}^{\mathcal{P}}+\Delta X_{t_{j}}^{\mathcal{P}}, \quad X_{t_{0}}^{\mathcal{P}}=0
$$

One then understands the limit of $X_{T}^{\mathcal{P}}$ for finer partitions as a mean-square limit,

$$
X_{t} \equiv x_{0}+\int_{0}^{t}\left\{v\left(X_{s}, s\right) d s+\sum_{\alpha} \sigma^{(\alpha)}\left(X_{s}, s\right) d M_{s}^{(\alpha)}\right\}:=\underset{\mid \mathcal{P} ! \rightarrow 0}{\lim . X_{t_{N}}^{\mathcal{P}}} .
$$

That is, $\lim _{\mid \mathcal{P} ! \rightarrow 0} \mathbb{E}\left[\left|X_{t}-X_{t_{N}}^{\mathcal{P}}\right|^{2}\right]=0$ if such a limit $X_{t}^{\mathcal{P}} \in L^{2}(\Omega, \mathcal{F}, \mathbb{P})$ exists. Amongst the various schemes to compute the increment $\Delta X_{t_{j}}^{\mathcal{P}}$, the simplest is the Euler method

$$
\Delta X_{t_{j}}^{\mathcal{P}}:=v\left(X_{t_{j}}^{\mathcal{P}}, t_{j}\right)\left[t_{j+1}-t_{j}\right]+\sum_{\alpha} \sigma^{(\alpha)}\left(X_{t_{j}}^{\mathcal{P}}, t_{j}\right)\left[M_{t_{j+1}}^{(\alpha)}-M_{t_{j}}^{(\alpha)}\right]
$$

an alternative is given by the averaging scheme

$$
\begin{aligned}
\Delta X_{t_{j}}^{\mathcal{P}}: & =\frac{1}{2}\left[v\left(X_{t_{j+1}}^{\mathcal{P}}, t_{j+1}\right)+v\left(X_{t_{j}}^{\mathcal{P}}, t_{j}\right)\right]\left[t_{j+1}-t_{j}\right] \\
& +\sum_{\alpha}\left[\sigma^{(\alpha)}\left(X_{t_{j+1}}^{\mathcal{P}}, t_{j+1}\right)+\sigma^{(\alpha)}\left(X_{t_{j}}^{\mathcal{P}}, t_{j}\right)\right]\left[M_{t_{j+1}}^{(\alpha)}-M_{t_{j}}^{(\alpha)}\right] .
\end{aligned}
$$

If the $\sigma^{(\alpha)}$ were zero, so that (2.1) reduces to an ODE, then the Euler scheme has local error of $O\left(h^{2}\right)$, whereas the averaging scheme has local error of $O\left(h^{3}\right)$, where $h$ is the largest increment size (which is, of course, proportional to $|\mathcal{P}|$ for the deterministic case). If the $\sigma^{(\alpha)}$ are nonzero then the noise increments $M_{t_{j+1}}-M_{t_{j}}$ may fluctuate rapidly enough to lead to $h=O\left(|\mathcal{P}|^{a}\right)$ with $a<1$. For instance, in the case of the Wiener process as noise one has $a=\frac{1}{2}$. In this situation the Euler scheme leads to an $0(|\mathcal{P}|)$ local error which makes a nontrivial contribution to the stochastic integral (2.3) which is not present in the averaging scheme. The respective solutions (when they exist) are called the Ito and 
Stratonovich stochastic integrals and the following notation shall be used: $X_{t}=X_{0}+\int_{0}^{t} d X_{s}$, with

$$
\begin{aligned}
\text { (Ito) } d X_{t} & =v\left(X_{t}, t\right) d t+\sum_{\alpha} \sigma^{(\alpha)}\left(X_{t}, t\right) d M_{t}^{(\alpha)}, \\
\text { (Stratonovich) } d X_{t} & =\overline{v\left(X_{t}, t\right)} d t+\sum_{\alpha} \overline{\sigma^{(\alpha)}\left(X_{t}, t\right)} d M_{t}^{(\alpha)} .
\end{aligned}
$$

The deterministic component of the integrand (coefficient of $d t$ ) is taken to be Riemann integrable and so the over- or under-bar notation can be omitted, as frequently will be the case. In general, for $X_{t}$ and $Y_{t}$ stochastic processes, one understands the limits $X_{t_{j}}, \quad=0$.

$$
\int_{0}^{t} X_{s} d Y_{s}:=\lim _{|\mathcal{P}| \rightarrow 0} \sum_{j=0}^{N-1} X_{t_{j}}^{\mathcal{P}} \Delta Y_{t_{j}}^{\mathcal{P}}, \quad \int_{0}^{t} \overline{X_{s}} d Y_{s}:=\underset{|\mathcal{P}| \rightarrow 0}{\lim .} \sum_{j=0}^{N-1} \overline{X_{t_{j}}^{\mathcal{P}}} \Delta Y_{t_{j}}^{\mathcal{P}},
$$

where $\Delta Y_{t_{j}}^{\mathcal{P}}:=Y_{t_{j+1}}^{\mathcal{P}}-Y_{t_{j}}^{\mathcal{P}}$ and $\overline{X_{t_{j}}^{\mathcal{P}}}:=\frac{1}{2}\left(X_{t_{j+1}}^{\mathcal{P}}-X_{t_{j}}^{\mathcal{P}}\right)$. Moreover, to compute the increments of the product $X, Y$, one notes

$$
\begin{aligned}
\Delta(X Y)_{t_{j}}^{\mathcal{P}} & =X_{t_{j+1}}^{\mathcal{P}} Y_{t_{j+1}}^{\mathcal{P}}-X_{t_{j}}^{\mathcal{P}} Y_{t_{j}}^{\mathcal{P}}=X_{t_{j}}^{\mathcal{P}} \Delta Y_{t_{j}}^{\mathcal{P}}+\Delta X_{t_{j}}^{\mathcal{P}} Y_{t_{j}}^{\mathcal{P}}+\Delta X_{t_{j}}^{\mathcal{P}} \Delta Y_{t_{j}}^{\mathcal{P}} \\
& =\overline{X_{t_{j}}^{\mathcal{P}}} \Delta Y_{t_{j}}^{\mathcal{P}}+\Delta X_{t_{j}}^{\mathcal{P}} \overline{Y_{t_{j}}^{\mathcal{P}}} .
\end{aligned}
$$

The terms $\Delta X \Delta Y$ need not be negligible; for the Wiener process it is $O(|\mathcal{P}|)$. Thus, if the noise processes $M_{t}^{(\alpha)}$ admit a stochastic calculus and if $X_{t}, Y_{t}$ are processes driven by these noises, then one expects that the Ito form breaks the Leibniz rule of calculus,

$$
d\left(X_{t} Y_{t}\right)=X_{t} d Y_{t}+d X_{t} Y_{t}+d X_{t} d Y_{t}
$$

while the Stratonovich form retains it

$$
d\left(X_{t} Y_{t}\right)=\overline{X_{t}} d Y_{t}+d X_{t} \overline{Y_{t}}
$$

here the equivalence relation means equality up to $O(d t)$ in the deterministic part which implies equality under the integral sign. Using (2.9b) inductively, one sees that

$$
d X_{t}^{2}=2 \overline{X_{t}} d X_{t}, \quad d X_{t}^{3}=\left(2{\overline{X_{t}}}^{2}+\overline{X_{t}^{2}}\right) d X_{t}, \quad d X_{t}^{4}=\left(\overline{X_{t}^{3}}+\overline{X_{t}} \overline{X_{t}^{2}}+2{\overline{X_{t}}}^{3}\right) d X_{t}, \quad \cdots
$$

Now $\overline{X_{t}^{n}}=\frac{1}{2}\left(X_{t}+d X_{t}\right)^{n}+\frac{1}{2} X_{t}^{n}$, from which it follows that $\overline{X_{t}^{n}}-{\overline{X_{t}}}^{n}=O\left(\left(d X_{t}\right)^{2}\right)$ and so

$$
\overline{X_{t}^{n}} d X_{t}={\overline{X_{t}}}^{n} d X_{t}+o\left(\left(d X_{t}\right)^{2}\right) \text {. }
$$

In the case of diffusion processes, one then has $\overline{X_{t}^{n}} d X_{t} \equiv{\overline{X_{t}}}^{n} d X_{t}$, and so $d X_{t}^{n} \equiv n{\overline{X_{t}}}^{n-1} d X_{t}$ which implies that the chain rule formula of standard calculus holds when applying the Stratonovich calculus to ditfusions. However, one can see already that this will not be true for processes driven by the Poisson process with nonlinear noise coefficients.

Examples Whereas the Stratonovich integral with respect to general martingales can be formulated, it is interesting to give explicitly the Wiener and Poisson cases.

Wiener Process as noise: The Wiener process $B_{t}$, admits a stochastic calculus with $\left(d B_{t}\right)^{2}=d t$ and higher powers $o(d t)$. The Stratonovich SDE $d X_{t}=v\left(X_{t}, t\right) d t+\overline{\sigma\left(X_{t}, t\right)} d B_{t}$ is well known to be equivalent to an Ito SDE $d X_{t}=$ $\tilde{v}\left(X_{t}, t\right) d t+\tilde{\sigma}\left(X_{t}, t\right) d B_{t}$, where the coefficients are related by

$$
\tilde{v}=v+\frac{1}{2} \sigma \sigma^{\prime}, \quad \tilde{\sigma}=\sigma,
$$

with $\sigma^{\prime}(x, t):=\frac{\partial}{\partial x} \sigma(x, t)$. Inversely, $v=\tilde{v}-\frac{1}{2} \tilde{\sigma}^{\prime} \tilde{\sigma}$.

Poisson process as noise: The Poisson process Nt admits a stochastic calculus with $\left(d N_{t}\right)^{n}=d N$, for all integers $n>{ }_{1}$. The fluctuations in $d N_{t}$ are $0(d t)$. The Stratonovich SDE $d X_{t}=\overline{v\left(X_{t}, t\right)} d t+\overline{\mu\left(X_{t}, t\right)} d N_{t}$, is equivalent to the Ito $\operatorname{SDE} d X_{t}=\tilde{v}\left(X_{t}, t\right) d t+\tilde{\mu}\left(X_{t}, t\right) d N_{t}$, where the coefficients are related by

$$
\tilde{v}=v, \quad \tilde{\mu}(x, t)=\frac{1}{2}[\mu(x+\tilde{\mu}(x, t))+\mu(x, t)] .
$$


The Stratonovich form for the Poisson integrals is next derived explicitly. Let $f=f(x)$ be analytic, then by Taylor expansion one has

$$
\begin{aligned}
f\left(X_{t_{j+1}}^{\mathcal{P}}\right)= & f\left(X_{t_{j}}^{\mathcal{P}}\right)+f^{\prime}\left(X_{t_{j}}^{\mathcal{P}}\right) \Delta X_{t_{j}}^{\mathcal{P}}+\frac{1}{2 !} f^{\prime \prime}\left(X_{t_{j}}^{\mathcal{P}}\right)\left(\Delta X_{t_{j}}^{\mathcal{P}}\right)^{2}+\cdots \\
\equiv & f\left(X_{t_{j}}^{\mathcal{P}}\right)+\left\{f^{\prime}\left(X_{t_{j}}^{\mathcal{P}}\right) \tilde{\mu}\left(X_{t_{j}}^{\mathcal{P}}, t_{j}\right)+\frac{1}{2 !} f^{\prime \prime}\left(X_{t_{j}}^{\mathcal{P}}\right) \tilde{\mu}\left(X_{t_{j}}^{\mathcal{P}}, t\right)+\cdots\right\} \Delta N_{t_{j}} \\
& +f^{\prime}\left(X_{t_{j}}^{\mathcal{P}}\right) \tilde{v}\left(X_{t_{j}}^{\mathcal{P}}, t_{j}\right) \Delta t_{j}+o\left(\Delta t_{j}\right) .
\end{aligned}
$$

Therefore, multiplying by $\Delta N_{t_{j}}=N_{t_{j+1}}-N_{t_{j}}$, and re-summing leads to

$$
f\left(X_{t_{j}}^{\mathcal{P}}\right) \Delta N_{t_{j}} \equiv f\left(X_{t_{j}}^{\mathcal{P}}+\tilde{\mu}\left(X_{t_{j}}^{\mathcal{P}}, t_{j}\right)\right) \Delta N_{t_{j}}
$$

On account of this,

$$
\begin{aligned}
X_{t_{j+1}}^{\mathcal{P}}= & X_{t_{j}}^{\mathcal{P}}+\frac{1}{2}\left\{v\left(X_{t_{j+1}}^{\mathcal{P}}, t_{j+1}\right)+v\left(X_{t_{j}}^{\mathcal{P}}, t_{j}\right)\right\} \Delta t_{j} \\
& +\frac{1}{2}\left\{\mu\left(X_{t_{j+1}}^{\mathcal{P}}, t_{j+1}\right)+\mu\left(X_{t_{j}}^{\mathcal{P}}, t_{j}\right)\right\} \Delta N_{t_{j}} \\
\equiv & X_{t_{j}}^{\mathcal{P}}+v\left(X_{t_{j}}^{\mathcal{P}}, t_{j}\right) \Delta t_{j}+\frac{1}{2}\left\{\mu\left(X_{t_{j}}^{\mathcal{P}}+\tilde{\mu}\left(X_{t_{j}}^{\mathcal{P}}, t_{j}\right), t_{j}\right)+\mu\left(X_{t_{j}}^{\mathcal{P}}, t_{j}\right)\right\} \Delta N_{t_{j}} .
\end{aligned}
$$

and so the relation (2.12) closes the transformation.

Remarks i) In the case of linear Poisson-noise coefficient $\mu(x)=\mu_{0} x+c$ the relation (2.12) gives $\tilde{\mu}(x)=\left(\mu_{0} x+c\right) /(l-$ $\left.\frac{1}{2} \mu_{0}\right)$. The requirement on the coefficients is that $x \mapsto x+\mu(x, t)$ is strictly monotone, for each $t$, and so defines a proper change of variable. For general $\mu(x)$ there exists no closed expression for $\tilde{\mu}$, however, it may be approximated by the iteration $\tilde{\mu}_{n+1}(x)=\frac{1}{2}\left\{\mu\left(x+\tilde{\mu}_{n}(x)\right)+\mu(x)\right\}, \mu_{0}(x)=\mu(x)$.

ii) There are other numerical schemes which can be employed. For instance, the mid-point 2nd order Runge-Kutta method

$$
X_{t_{j+1}}^{\mathcal{P}}=X_{t_{j}}^{\mathcal{P}}+v\left(\tilde{X}_{t_{j}^{*}}^{\mathcal{P}}, t_{j}^{*}\right) \Delta t_{j}+\sum_{\alpha} \sigma^{(\alpha)}\left(\tilde{X}_{t_{j}^{*}}^{\mathcal{P}}, t_{j}^{*}\right) \Delta M_{t_{j}}^{(\alpha)}
$$

where $t_{j}^{*}:=\frac{1}{2}\left(t_{j+1}+t_{j}\right)$ and $\tilde{X}_{t_{j}^{*}}^{\mathcal{P}}$ is obtained from an Euler time-step from $t_{j}$ to $t_{j}^{*}$

$$
\tilde{X}_{t_{j}^{*}}^{\mathcal{P}}=X_{t_{j}}^{\mathcal{P}}+v\left(\tilde{X}_{t_{j}^{*}}^{\mathcal{P}}, t_{j}^{*}\right)\left[t_{j}^{*}-t_{j}\right]+\sum_{\alpha} \tilde{\sigma}^{(\alpha)}\left(X_{t_{j}}^{\mathcal{P}}, t_{j}^{*}\right)\left[M_{t_{j}^{*}}^{(\alpha)}-M_{t_{j}}^{(\alpha)}\right]
$$

with $\tilde{v}, \tilde{\sigma}^{(\alpha)}$ the associated Ito coefficients. This procedure is, in fact, equivalent to the Stratonovich choice. However, the method given by is equivalent to the Stratonovich choice only for the Wiener process as noise. For the Poisson process, it coincides with the Stratonovich choice only if $\mu(x, t)$ is linear in $x$; otherwise it leads to a stochastic integral equivalent to an Ito integral with coefficient $\hat{\mu}(x, t)$ related by $\mu\left(x+\frac{1}{2} \hat{\mu}(x, t), t\right)=\hat{\mu}(x, t)$.

iii) In general $X_{t}$ is said to be Riemann integrable with respect to $Y_{t}$, if the mean-square limit of $\sum_{j} X_{s_{j}}^{\mathcal{P}} \Delta Y_{t_{j}}^{\mathcal{P}}$ exists whenever an arbitrary prescription to determine $s \in\left[t_{j}, t_{j+l}\right]$ is given. The Ito calculus is based on the choice $s_{j}=t_{j}$. One may call this the retarded Ito theory. The advanced Ito theory can be defined via the prescription that $s_{j}=$ $t_{j+l}$. The Stratonovich calculus then corresponds to the averaging over the retarded and advanced Ito versions. An alternative definition of Stratonovich integrals as simply mean-square Riemann integrals with $s_{j}=t_{j}^{*}$ is frequently taken in probabilistic literature, however, as seen in the previous remark this does not always coincide with the definition here.

\section{Quantum Stochastic Calculus}

We begin by recalling the Hudson-Parthasarathy theory of noncommutative stochastic processes [12, 20]. Let $\mathcal{H}_{0}$ and $\mathcal{K}$ be fixed Hilbert spaces called the initial and internal spaces, respectively. $L^{2}(I ; \mathcal{K}) \cong L^{2}(I) \otimes \mathcal{K}$ is the Hilbert space of square-integrable $\mathcal{K}$-valued functions on an interval $I \subset \mathbb{R}^{+}$. The (Bose) Fock space over $L^{2}(I ; \mathcal{K})$ is denoted by $\Gamma_{+}(I ; \mathcal{K})$. In the following, a quantum stochastic process will be understood as an operator-valued family $\left(X_{t}\right)_{t \geq 0}$ on $\mathcal{H}_{0}$ 
$\otimes \Gamma_{+}\left(\mathbb{R}^{+}, \mathcal{K}\right)$. The initial value of the process $X_{0}$ will be taken as an element $x_{0} \in \mathcal{B}\left(\mathcal{H}_{0}\right)$. The noise space admits the following continuous tensor-product decomposition,

$$
\Gamma_{+}\left(\mathbb{R}^{+}, \mathcal{K}\right) \cong \Gamma_{+}([0, t] ; \mathcal{K}) \otimes \Gamma_{+}((t, \infty) ; \mathcal{K})
$$

which allows the introduction of a time filtration. In particular, a process $\left(X_{t}\right)_{t \geq 0}$ is adapted if, for each $t>0, X_{t} \varphi=\varphi$, for all $\varphi \in \Gamma_{+}((t, \infty) ; \mathcal{K})$.

Let $\Psi(f)$ denote the exponential vector with test function $f \in L^{2}\left(\mathbb{R}^{+}, \mathcal{K}\right)$ and for some subset $\mathcal{S}$ let $\operatorname{EXP}(\mathcal{S})$ be the $\operatorname{span}$ of exponential vectors with test functions in $\mathcal{S}$; the conserver $\Lambda(M)$, creator $A^{\dagger}(g)$ and destroyer $A(g)$ are operators on $\Gamma_{+}\left(\mathbb{R}^{+}, \mathcal{K}\right)$ defined by their actions on the span of exponential vectors,

$$
\begin{aligned}
\Lambda(M) \Psi(f): & =-\left.i \frac{\partial}{\partial \varepsilon} \Psi\left(e^{i \varepsilon M} f\right)\right|_{\varepsilon=0}, \\
A(g) \Psi(f): & =\left.\frac{\partial}{\partial \varepsilon} \Psi(f+\varepsilon g)\right|_{\varepsilon=0}, \\
A^{\dagger}(g) \Psi(f) & :=\langle g, f\rangle \Psi(g) .
\end{aligned}
$$

The following notation will be used here

$$
A_{t}^{11}:=\Lambda\left(P_{[0, t]}\right), A_{t}^{10}(g):=A^{\dagger}\left(\chi_{[0, t]} \otimes g\right), A_{t}^{01}(g):=A\left(\chi_{[0, t]} \otimes g\right), A_{t}^{00}:=t
$$

for $t \geq 0, g \in L_{\mathrm{loc}}^{\infty}\left(\mathbb{R}^{+} ; \mathcal{K}\right)$ and $P_{[0, t]}: f \mapsto \chi_{[0,1]} \otimes f$. They are called the conserver, creator, destroyer and standard time process, respectively. One may write $A_{t}^{11}, A_{t}^{10}, A_{t}^{01}, A_{t}^{00}$ for the creator and destroyer when the intensity $g \equiv 1$. For general $g$ one then has $A_{t}^{10}(g)=\int_{0}^{t} g(s) d A_{s}^{10}$ and $A_{t}^{01}(g)=A_{t}^{10}(g)^{\dagger}$.

Let $\mathcal{D}$ be a linear domain in $\mathcal{H}_{0}$ and let $\mathcal{S}$ be a dense linear manifold (called an admissible space) in $L^{2}\left(\mathbb{R}^{+} ; \mathcal{K}\right)$ closed under the action of the projections $P_{[0, t]},(t \geq 0)$. An adapted process $\left(X_{t}\right)_{t \geq 0}$ is said to be based on $(\mathcal{D}, \mathcal{S})$ if each $X_{t}$, is the ampliation to $\mathcal{D} \otimes \operatorname{EXP}\left(P_{[0, t]} \mathcal{S}\right) \otimes \Gamma_{+}((t, \infty) ; \mathcal{K})$ of an operator on the domain $\mathcal{D} \otimes \operatorname{EXP}\left(P_{[0, t]} \mathcal{S}\right)$.

Hudson and Parthasarathy [12] define stochastic integrals of the type

$$
\begin{aligned}
X_{t} \quad: & =x_{0}+\int_{0}^{t}\left\{X_{s}^{11} \otimes d A_{s}^{11}+X_{s}^{10} \otimes d A_{s}^{10}+X_{s}^{01} \otimes d A_{s}^{01}+X_{s}^{00} \otimes d A_{s}^{00}\right\} \\
& =x_{0}+\int_{0}^{t} X_{s}^{\alpha \beta} \otimes d A_{s}^{\alpha \beta}
\end{aligned}
$$

where the $\left(X_{t}^{\alpha \beta}\right)_{t \geq 0}$ are adapted quantum stochastic processes based on $(\mathcal{D}, \mathcal{S})$ which are weakly measurable and satisfy the local square-integrability conditions below,

$$
\int_{0}^{t} d s|f(s)|\left\|X_{s}^{11} u \underline{\otimes} \Psi(f)\right\|^{2}<\infty, \int_{0}^{t} d s\left\|X_{s}^{\alpha \beta} u \underline{\otimes} \Psi(f)\right\|^{2}<\infty,
$$

for arbitrary $t>0, u \in \mathcal{D}$ and $f \in \mathcal{S}$.

The notation in (3.4) disguises the fact that the integrators commute with the integrands (when adapted). The algebraic manipulation of stochastic integrals is then summarized by the quantum Ito formula for adapted quantum stochastic processes $X_{t}$ and $Y_{t}$,

$$
d\left(X_{t} Y_{t}\right)=X_{t} d Y_{t}+d X_{t} Y t+d X_{t} d Y_{t}
$$

where the last term is the quantum Ito correction and can be evaluated using the quantum Ito table

$$
d A_{t}^{\alpha 1} d A_{t}^{1 \beta}=d A_{t}^{\alpha \beta} \text {, all other products vanishing. }
$$

DEFINITION 3.1. Let $X_{t}$ and $Y_{t}$ be adapted processes. One defines Stratonovich quantum stochastic integrals as

$$
\int \overline{X_{t}} d Y_{t}:=\int\left(X_{t}+\frac{1}{2} d X_{t}\right) d Y_{t} ; \int d X_{t} \overline{Y_{t}}:=\int d X_{t}\left(Y_{t}+\frac{1}{2} d Y_{t}\right)
$$


The product formula is then $X_{t} Y_{t}=x_{0} y_{0}+\int_{0}^{t}\left(d X_{s} \overline{Y_{s}}+\overline{X_{s}} d Y_{s}\right)$. The integrands $\overline{X_{t}}, \overline{Y_{t}}$ do not commute with the differentials $d X_{t}, d Y_{t}$ under the integral sign. This lack of commutativity will result in Stratonovich integrals having left and right hand forms. To work out general transformations between the two calculi for functions of a process, the following lemma will be useful. It is easily established by induction.

LEMMA 3.2. If $X_{t}$ satisjies the QSDE $d X_{t}=X_{t}^{\alpha \beta} \otimes d A_{t}^{\alpha \beta}$ then, for polynomial $f=f(x)=\sum f_{n} x^{n}$, the quantum Ito calculus gives

$$
d f\left(X_{t}\right)=f\left(X_{t}+d X_{t}\right)-f\left(X_{t}\right)=f\left(X_{t}\right)^{\alpha \beta} \otimes d A_{t}^{\alpha \beta},
$$

where

$$
\begin{aligned}
f\left(X_{t}\right)^{11}= & f\left(X_{t}+X_{t}^{11}\right)-f\left(X_{t}\right), \\
f\left(X_{t}\right)^{10}= & \sum_{n} f_{n} \sum_{p_{1}, p_{2}}^{n}\left(X_{t}+X_{t}^{11}\right)^{p_{1}} X_{t}^{10}\left(X_{t}\right)^{p_{2}}, \\
f\left(X_{t}\right)^{01}= & \sum_{n} f_{n} \sum_{p_{1}, p_{2}}^{n}\left(X_{t}\right)^{p_{1}} X_{t}^{01}\left(X_{t}+X_{t}^{11}\right)^{p_{2}}, \\
f\left(X_{t}\right)^{00}= & \sum_{n} f_{n} \sum_{p_{1}, p_{2}}^{n}\left(X_{t}\right)^{p_{1}} X_{t}^{00}\left(X_{t}\right)^{p_{2}} \\
& +\sum_{n} f_{n} \sum_{p_{1}, p_{2}, p_{3}}^{n}\left(X_{t}\right)^{p_{1}} X_{t}^{01}\left(X_{t}+X_{t}^{11}\right)^{p_{2}} X_{t}^{10}\left(X_{t}\right)^{p_{3}},
\end{aligned}
$$

Now, from the definition $\overline{f\left(X_{t}\right)} d A_{t}^{\alpha \beta}=\left[f\left(X_{t}\right)+\frac{1}{2} d f\left(X_{t}\right)\right] d A_{t}^{\alpha \beta}$ and with the use of the quantum Ito table, one has

$$
\begin{aligned}
\overline{f\left(X_{t}\right)} d A_{t}^{11} & =\frac{1}{2}\left[f\left(X_{t}+X_{t}^{11}\right)+f\left(X_{t}\right)\right] d A_{t}^{11}+\frac{1}{2} f\left(X_{t}\right)^{01} d A_{t}^{01}, \\
\overline{f\left(X_{t}\right)} d A_{t}^{10} & =\frac{1}{2}\left[f\left(X_{t}+X_{t}^{11}\right)+f\left(X_{t}\right)\right] d A_{t}^{10}+\frac{1}{2} f\left(X_{t}\right)^{01} d A_{t}^{00}, \\
\overline{f\left(X_{t}\right)} d A_{t}^{01} & =f\left(X_{t}\right) d A_{t}^{01}, \\
\overline{f\left(X_{t}\right)} d A_{t}^{00} & =f\left(X_{t}\right)^{00} d A_{t}^{00} .
\end{aligned}
$$

Likewise

$$
\begin{aligned}
d A_{t}^{11} \overline{f\left(X_{t}\right)} & =\frac{1}{2}\left[f\left(X_{t}+X_{t}^{11}\right)+f\left(X_{t}\right)\right] d A_{t}^{11}+\frac{1}{2} f\left(X_{t}\right)^{01} d A_{t}^{01}, \\
d A_{t}^{10} \overline{f\left(X_{t}\right)} & =f\left(X_{t}\right) d A_{t}^{10}, \\
d A_{t}^{01} \overline{f\left(X_{t}\right)} & =\frac{1}{2}\left[f\left(X_{t}+X_{t}^{11}\right)+f\left(X_{t}\right)\right] d A_{t}^{01}+\frac{1}{2} f\left(X_{t}\right)^{10} d A_{t}^{00}, \\
d A_{t}^{00} \overline{f\left(X_{t}\right)} & =f\left(X_{t}\right)^{00} d A_{t}^{00} .
\end{aligned}
$$

Remember though that, as in the classical situation, these notations apply only under the integral sign and are strictly nonassociative; that is, $\overline{X_{t} Y_{t}} d Z_{t} \neq \overline{X_{t}} \overline{Y_{t}} d Z_{t}$.

THEOREM 3.3. The following transformations exist between QSDEs where the coefficients are related according to (2.11) and (2.12), respectively,

$$
\begin{aligned}
(i) \quad d X_{t} & =\overline{v\left(X_{t}, t\right)} d t+\overline{\sigma\left(X_{t}, t\right)}\left\{A_{t}^{10}+d A_{t}^{10}\right\} \\
& =\tilde{v}\left(X_{t}, t\right) d t+\tilde{\sigma}\left(X_{t}, t\right)\left\{A_{t}^{10}+d A_{t}^{10}\right\} \\
& =d t \overline{v\left(X_{t}, t\right)} d t+\left\{A_{t}^{01}+d A_{t}^{01}\right\} \overline{\sigma\left(X_{t}, t\right)} \\
(\text { ii }) \quad d X_{t} & =\overline{v\left(X_{t}, t\right)} d t+\overline{\mu\left(X_{t}, t\right)}\left\{d A_{t}^{11}+d A_{t}^{10}+d A_{t}^{10}+d A_{t}^{00}\right\} \\
& =\tilde{v}\left(X_{t}, t\right) d t+\tilde{\mu}\left(X_{t}, t\right)\left\{d A_{t}^{11}+d A_{t}^{10}+d A_{t}^{10}+d A_{t}^{00}\right\} \\
& =d t \overline{v\left(X_{t}, t\right)}+\left\{d A_{t}^{11}+d A_{t}^{10}+d A_{t}^{10}+d A^{00}\right\} \overline{\mu\left(X_{t}, t\right)}
\end{aligned}
$$


Proof. In (i), the process $A_{t}^{10}+A_{t}^{01}$ in the Fock vacuum state, corresponds to the Wiener process. Here $X_{t}^{11}=0$, $X_{t}^{01}=X_{t}^{10}=\tilde{\sigma}\left(X_{t}, t\right)$ and $X_{t}^{00}=\tilde{v}\left(X_{t}, t\right)$. From (3.11) one has that the reordering of $d A_{t}^{10}$ will lead to the Stratonovich QSDE with integrators to the right being related to the Ito QSDE by

$$
\overline{f\left(X_{t}\right)} d A_{t}^{10}=f\left(X_{t}\right) d A_{t}^{10}+\frac{1}{2} \sum_{n} f_{n} n\left(X_{t}\right)^{n-1} \tilde{\sigma}\left(X_{t}, t\right) d A_{t}^{00},
$$

the last term is clearly just $\frac{1}{2} f^{\prime}\left(X_{t}\right) \tilde{\sigma}\left(X_{t}, t\right) d t$ and so

$$
\overline{\tilde{\sigma}\left(X_{t}, t\right)} d A_{t}^{10}=\tilde{\sigma}\left(X_{t}, t\right) d A_{t}^{10}+\frac{1}{2} \tilde{\sigma}^{\prime}\left(X_{t}, t\right) \tilde{\sigma}\left(X_{t}, t\right) d A_{t}^{00}
$$

This establishes the relationship. The alternative Stratonovich QSDE where the noise coefficients lie to the right of the noise is handled with (3.12).

In (ii), the process $A_{t}^{11}+A_{t}^{10}+A_{t}^{10}+A_{t}^{00}$, in the Fock vacuum state, corresponds to the Poisson process. Here $X_{t}^{11}=$ $X_{t}^{10}=X_{t}^{10}=X_{t}^{00}-\tilde{v}\left(X_{t}, t\right)=\tilde{\mu}\left(X_{t}, t\right)$. To obtain the Stratonovich QSDE with integrators on the right, the terms leading to differences are those in $d A^{11}$ and $d A^{10}$; from (3.11) again one has

$$
\begin{aligned}
\overline{f\left(X_{t}\right)}\left\{d A_{t}^{11}+A_{t}^{10}\right\}= & \frac{1}{2}\left\{f\left(X_{t}+\tilde{\mu}\right)+f\left(X_{t}\right)\right\}\left\{d A_{t}^{11}+A_{t}^{10}\right\} \\
& +\frac{1}{2} \sum_{n} f_{n} \sum_{p_{1}, p_{2}}^{n}\left(X_{t}\right)^{p_{1}} \tilde{\mu}\left(X_{t}+\tilde{\mu}\right)^{p_{2}}\left\{d A_{t}^{01}+A_{t}^{00}\right\} .
\end{aligned}
$$

The coefficient of the last term sums to $\frac{1}{2}\left\{f\left(X_{t}+\tilde{\mu}\left(X_{t}, t\right)\right)-f\left(X_{t}\right)\right\}$, and so one concludes

$$
\begin{aligned}
& \overline{f\left(X_{t}\right)}\left\{d A_{t}^{11}+d A_{t}^{10}+d A_{t}^{10}+d A_{t}^{00}\right\} \\
& +\frac{1}{2}\left\{f\left(X_{t}+\tilde{\mu}\left(X_{t}, t\right)\right)-f\left(X_{t}\right)\right\}\left\{d A_{t}^{11}+d A_{t}^{10}+d A_{t}^{10}+d A_{t}^{00}\right\} .
\end{aligned}
$$

\section{Quantum white noise representation}

There is a more natural way to look at this problem. Take as admissible space the subset $\mathcal{S} \subset L^{2}\left(\mathbb{R}^{+}, \mathcal{K}\right)$ got by taking the sup-norm completion of the square-integrable step functions on $\mathbb{R}^{+}$. That is, $\mathrm{S}$ is the set of square-integrable regulated functions and for $f \in \mathcal{S}$ one is guaranteed that the past and future instant limits $f\left(t^{-}\right)$and $f\left(t^{+}\right)$exist at each $t>0$, cf. Dieudonne [21]. Note that the projection requirement of admissible spaces rules out the Schwartz functions; the space of test functions $\mathcal{S}$ is in a sense the most natural choice of it as the widest space on which integral approximations can be based and also contains the functions of bounded variation which are the natural space on which to discuss functional integral transforms. One defines functionals $\delta_{ \pm}(t)$ and $\delta_{*}(t)$ on $\mathcal{S}$ by

$$
\left\langle\delta_{ \pm}(t), f\right\rangle=f\left(t^{ \pm}\right), \quad\left\langle\delta_{*}(t), f\right\rangle=\frac{1}{2}\left\{f\left(t^{+}\right)+f\left(t^{-}\right)\right\} .
$$

That is, the action of $\delta_{*}(t)$ on a function of a time variable is to give the average of the immediate past and future values at time $t$. The action of these functionals can be extended by linearity from $\mathcal{S}$ to $\mathcal{S}^{\prime}$ if the following identifications are made:

$$
\begin{aligned}
& \left\langle\delta_{-}(t), \delta_{+}(s)\right\rangle:=\delta_{+}(t-s), \\
& \left\langle\delta_{+}(t), \delta_{-}(s)\right\rangle:=\delta_{-}(t-s), \\
& \left\langle\delta_{+}(t), \delta_{+}(s)\right\rangle:=\delta_{*}(t-s), \\
& \left\langle\delta_{-}(t), \delta_{-}(s)\right\rangle:=\delta_{*}(t-s),
\end{aligned}
$$

DEFINITION 4.1. A basic step function on $\mathbb{R}^{n}$ is the characteristic function of a set of the form $\left\{\left(t_{1}, \cdots, t_{n}\right): 0 \leq t_{j(1)}-c_{1} \leq \cdots \leq\right.$ where $1 \leq r \leq n$, the $j(l), \ldots, j(r)$ are distinct elements of $\{1, \ldots, n\}$, and $c_{0}, c_{1}, \cdots, c_{r}$ are constants, and also where any 
of the strict inequalities can be replaced by ordinary ones. A simple function on $\mathbb{R}^{n}$ is a finite linear combination of such step functions. The space of multi-dimensional regulated functions $\mathcal{S}_{n}$, is taken to be the sup-norm completion of these simple functions.

For $f \in \mathcal{S}$, the individual limits of $f\left(t_{1}, \cdots, t_{n}\right)$ exist as the $t_{j} \rightarrow a_{j}^{ \pm}$under the conditions $t_{\sigma(1)}<\cdots<t_{\sigma(n)}$ for all $a_{j} \in \mathbb{R}$, and for each permutation $\sigma$ of $\{1, \cdots, n\}$.

DEFINITION 4.2. Quantum white noises are defined on $\operatorname{EXP}(\mathcal{S})$ by $a_{ \pm}^{\sharp}(t):=A^{\sharp}\left(\delta_{ \pm}(t)\right)$ and $a_{*}^{\sharp}(t):=A^{\sharp}\left(\delta_{*}(t)\right)$. Explicitly, for $f \in \mathcal{S}$, one has $a_{ \pm}(t) \Psi(f)=f\left(t^{ \pm}\right) \Psi(f)$, etc.

From (4.2), the nontrivial commutations between the $a_{ \pm}^{\sharp}(t)$ are given by

$$
\begin{aligned}
& {\left[a_{-}(t), a_{+}^{\dagger}(s)\right]=\delta_{+}(t-s),} \\
& {\left[a_{+}(t), a_{-}^{\dagger}(s)\right]=\delta_{-}(t-s),} \\
& {\left[a_{+}(t), a_{+}^{\dagger}(s)\right]=\delta_{*}(t-s),} \\
& {\left[a_{-}(t), a_{-}^{\dagger}(s)\right]=\delta_{*}(t-s),}
\end{aligned}
$$

The linearity of the extended functionals then implies that the pair of processes $\left\{a_{*}^{\sharp}(t) ; t>0\right\}$ satisfy the following canonical commutation relations $(\mathrm{CCR})$

$$
\left[a_{*}(t), a_{*}(s)\right]=0=\left[a_{*}^{\dagger}(t), a_{*}^{\dagger}(s)\right], \quad\left[a_{*}(t), a_{*}^{\dagger}(s)\right]=\delta_{*}(t-s) .
$$

One further has the following functional distribution

$$
\left\langle\exp \left(\int{ }_{0}^{\infty} d t\left\{f(t) a_{*}^{\dagger}(t)+f(t)^{*} a_{*}^{\dagger}(t)\right\}\right)\right\rangle=\exp \left[-\frac{1}{2}\|f\|^{2}\right],
$$

where $f \in \mathcal{S}$, and $\|f\|^{2}=\int_{0}^{\infty}|f(t)|^{2} d t$, and the expectation is in the Fock vacuum state $\Psi(0)$. The key feature of (4.4) is that, along with the specification of the state (4.5), it contains all information concerning the chaotic expansions. Any integral of the form $\int_{\mathbb{R}^{n}} d t_{1} \cdots d t_{n} \varphi\left(t_{1}, \cdots, t_{n}\right) a_{*}^{\sharp(1)}\left(t_{1}\right) \cdots a_{*}^{\sharp(n)}\left(t_{n}\right)$, for $\varphi \in \mathcal{S}$, can be evaluated and, in particular, one may take $\varphi$ to be simplicial.

The following connection $[10,17]$ exists between the quantum stochastic calculus and white noise calculus.

THEOREM 4.3. Let $\left(X_{t}\right)_{t \geq 0}$ be the solution of the QSDE $d X_{t}=X_{t}^{\alpha \beta} \otimes d A_{t}^{\alpha \beta}$ with the $\left(X_{t}^{\alpha \beta}\right)_{t \geq 0}$ adapted processes based on $(\mathcal{D}, \mathcal{S})$, then the QSDE can be represented as

$$
\begin{aligned}
d X_{t} & =\left\{a_{*}^{\dagger}(t) X_{t}^{11} a_{*}(t)+a_{*}^{\dagger}(t) X_{t}^{10}+X_{t}^{01} a_{*}(t)+X_{t}^{00}\right\} d t \\
& =\left[1, a_{*}^{\dagger}(t)\right]\left[\begin{array}{cc}
X_{t}^{00} & X_{t}^{01} \\
X_{t}^{10} & X_{t}^{11}
\end{array}\right]\left[\begin{array}{c}
1 \\
a_{*}(t)
\end{array}\right]
\end{aligned}
$$

\section{Remarks}

i) The Wiener and Poisson processes are represented by $B_{t}=\int_{0}^{t}\left(a_{*}^{\dagger}(s)+a_{*}(s)\right) d s, N_{t}=\int_{0}^{t}\left(1+a_{*}(s)\right)^{\dagger}\left(1+a_{*}(s)\right) d s$, respectively. Their chaotic expansions can readily be obtained.

ii) A Stratonovich QSDE is an equation of the form

$$
\begin{aligned}
d X_{t} & =\left\{a_{*}^{\dagger}(t) a_{*}(t){\overline{E_{t}}}^{R}+a_{*}^{\dagger}(t){\overline{F_{t}}}^{R}+a_{*}(t){\overline{G_{t}}}^{R}+{\overline{H_{t}}}^{R}\right\} d t \\
d X_{t} & =\left\{{\overline{E_{t}}}^{L} a_{*}^{\dagger}(t) a_{*}(t)+{\overline{F_{t}}}^{L} a_{*}^{\dagger}(t)+\bar{G}_{t}^{L} a_{*}(t)+{\overline{H_{t}}}^{L}\right\} d t
\end{aligned}
$$

Equation (4.7a) is the left handed version, and (4.7b) is the right handed version.

If the conserver terms are ignored, then one sees that the quantum Ito calculus corresponds to the Wick ordering scheme while the quantum Stratonovich corresponds to the Weyl scheme. This point of view can be of help in understanding the related distinctions which arise in the theory of phase space path integrals [22]. The anti-Wick ordering scheme gives time-reversed quantum Brownian motion.

iii) The classical Wiener integral is then represented as

$$
X_{t}=X_{0}+\int_{0}^{t}\left\{\overline{v\left(X_{s}, s\right)}+\left[a_{*}^{\dagger}(s)+a_{*}(s)\right] \overline{\sigma\left(X_{s}, s\right)}\right\} d s
$$




$$
X_{t}=X_{0}+\int_{0}^{t}\left\{\underline{\tilde{v}\left(X_{s}, s\right)}+a_{*}^{\dagger}(s) \underline{\tilde{\sigma}\left(X_{s}, s\right)}+\underline{\tilde{\sigma}\left(X_{s}, s\right)} a_{*}(s)\right\} d s
$$

where $v, \sigma$ and $\tilde{v}, \tilde{\sigma}$ are related by (2.11).

Likewise the classical Poissonian integral is represented as

$$
X_{t}=X_{0}+\int_{0}^{t}\left\{\overline{v\left(X_{s}, s\right)}+\left[1+a_{*}(s)\right]^{\dagger}\left[1+a_{*}(s)\right] \overline{\mu\left(X_{s}, s\right)}\right\} d s
$$

or

$$
X_{t}=X_{0}+\int_{0}^{t}\left\{\underline{\tilde{v}\left(X_{s}, s\right)}+\left[1+a_{*}(s)\right]^{\dagger} \underline{\tilde{\mu}\left(X_{s}, s\right)}\left[1+a_{*}(s)\right]\right\} d s
$$

where $v, \mu$ and $\tilde{v}, \tilde{\mu}$ are related by $(2.12)$.

iv) Let $X_{t}=x_{0}+\int_{0}^{t} X_{t}^{\alpha \beta} \otimes d A_{t}^{\alpha \beta}, Y_{t}=y_{0}+\int_{0}^{t} Y_{t}^{\alpha \beta} \otimes d A_{t}^{\alpha \beta}$ be processes based on $(\mathcal{D}, \mathcal{S})$, then

$$
\begin{aligned}
\int_{0}^{t} \overline{X_{s}} d Y_{s}= & \int_{0}^{t} d s\left(x_{0}+\int_{0}^{s} d s\left\{a_{*}^{\dagger}(s) X_{s}^{11} a_{*}(s)+a_{*}^{\dagger}(s) X_{s}^{10}+X_{s}^{01} a_{*}(s)+X_{s}^{00}\right\}\right) \\
& \times\left\{a_{*}^{\dagger}(s) Y_{s}^{11} a_{*}(s)+a_{*}^{\dagger}(s) Y_{s}^{10}+Y_{s}^{01} a_{*}(s)+Y_{s}^{00}\right\} \\
= & \int_{0}^{t} d s\left\{a_{*}^{\dagger}(s) X_{s} Y_{s}^{11} a_{*}(s)+a_{*}^{\dagger}(s) X_{s} Y_{s}^{10}+X_{s} Y_{s}^{01} a_{*}(s)+X_{s} Y_{s}^{00}\right\} \\
& +\int_{0}^{t} d s \int_{0}^{s} d u \delta_{*}(u-s)\left[a_{*}^{\dagger}(u) X_{u}^{11} Y_{s}^{11} a_{*}(s)\right. \\
& \left.+a_{*}^{\dagger}(u) X_{u}^{11} Y_{s}^{10}+X_{u}^{01} Y_{s}^{11} a_{*}(s)+X_{u}^{01} Y_{s}^{10}\right] \\
= & \int_{0}^{t} X_{s} d Y_{s}+\frac{1}{2} \int_{0}^{t} d s\left[X_{s}^{01}+a_{*}^{\dagger}(s) X_{s}^{11}\right]\left[Y_{s}^{10}+Y_{s}^{11} a_{*}(s)\right] .
\end{aligned}
$$

That is, $\int_{0}^{t} \overline{X_{s}} d Y_{s}=\int_{0}^{t} X_{s} d Y_{s}+\frac{1}{2} \int_{0}^{t} d X_{s} d Y_{s}$.

Thus the algebraic product $X_{t} Y_{t}$ of the white noise representations gives the correct product as quantum stochastic processes.

v) From the above considerations, it follows that the following formal manipulations are allowed

$$
\begin{aligned}
\int_{0}^{T} X_{t} \otimes d A_{t}^{01}-\int_{0}^{T} d A_{t}^{01} \overline{X_{t}} & =\int_{0}^{T}\left[X_{t}, a_{*}(t)\right] d t \\
& =\int_{0}^{T} d t \int_{0}^{t} d s\left[a_{*}^{\dagger}(t) X_{t}^{11} a_{*}(t)+a_{*}^{\dagger}(t) X_{t}^{10}+X_{t}^{01} a_{*}(t)+X_{t}^{00}, a_{*}(s)\right] \\
& =-\int_{0}^{T} d t \int_{0}^{t} d s \delta_{*}(t-s)\left[X_{s}^{11} a_{*}(s)+X_{s}^{10}\right] \\
& =-\frac{1}{2} \int_{0}^{T} d t\left[X_{t}^{11} a_{*}(t)+X_{t}^{10}\right] \\
& =-\frac{1}{2} \int_{0}^{T} d t\left[X_{t}^{11} \otimes d A_{t}^{10}+X_{t}^{10} \otimes d A_{t}^{00}\right] .
\end{aligned}
$$

More generally, for polynomial $f$,

$$
d f\left(X_{t}\right)=\left\{a_{*}^{\dagger}(t) f\left(X_{t}\right)^{11} a_{*}(t)+a_{*}^{\dagger}(t) f\left(X_{t}\right)^{10}+f\left(X_{t}\right)^{01} a_{*}(t)+f\left(X_{t}\right)^{00}\right\} d t
$$

and formal manipulation leads to

$$
\left[f\left(X_{t}\right), a_{*}(t)\right]=-\frac{1}{2} f\left(X_{t}\right)^{11} a_{*}(t)-\frac{1}{2} f\left(X_{t}\right)^{10},
$$

or under rearrangement

$$
\frac{1}{2} f\left(X_{t}+X_{t}^{11}\right)+f\left(X_{t}\right) a_{*}(t)=a_{*}(t) \overline{f\left(X_{t}\right)}-\frac{1}{2} f\left(X_{t}\right)^{10} .
$$

This is the same as the third relation of (3.12). 


\section{$5 \quad$ Asymptotic quantum stochastic limits; convergence ansatz}

Let $\mathcal{K}_{0}$ be a fixed Hilbert space and $S(t)=e^{i \Omega t}$ be a strongly continuous one-parameter unitary group on $\mathcal{K}_{0}$. If $\tilde{\mathcal{K}}_{0}$ is the subspace of $\mathcal{K}_{0}$ such that $\int_{-\infty}^{\infty} d t|\langle S(t) g, f\rangle|<\infty$ whenever $f, g \in \mathcal{K}_{0}$, then introducing the sesquilinear form

$$
\gamma(f, g)=\int_{-\infty}^{\infty} d t\langle S(t) g, f\rangle
$$

the Hilbert space completion of $\tilde{\mathcal{K}}_{0}$, with subspace $\{k: \gamma(k, k)=0\}$ factored out, will be denoted by $\mathcal{K}$. The inner product on $\mathcal{K}$ will be taken to be that inherited from $\mathcal{K}_{0}$ which will be denoted $\langle\cdot, \cdot\rangle_{\mathcal{K}}$. For each $g \in \mathcal{K}$ the mapping $t \rightarrow S(t) g$ is Bochner integrable. The following operator is well defined on $\mathcal{K}$ :

$$
S_{+}:=\int_{0}^{\infty} S(t) d t \equiv \frac{i}{\Omega+i 0^{+}}=\pi \delta(\Omega)+i P V \frac{1}{\Omega}
$$

and the notation $S_{-}:=\left(S_{+}\right)^{\dagger}, \Gamma=2 \operatorname{Re} S_{+}$and $\Sigma:=\operatorname{Im} S_{+}$shall be adopted. Noting that $\Gamma \geq 0$ and that $[\Gamma, \Sigma]=0$, it follows that there exists $Z=X+i Y$ with $X$ and $Y$ self-adjoint on $\mathcal{K}$ such that

$$
X^{2}=\frac{1}{4} \Gamma, \quad X Y+Y X=-\Sigma .
$$

For each $\gamma>0$, one considers $\mathcal{H}_{\lambda}$ a copy of $\Gamma_{+}(\mathcal{K})$ with Fock vacuum vector denoted as $\Psi_{\lambda}$ and $A_{\lambda}^{\sharp}(\cdot)$ the creator/destroyer maps. For $g \in \mathcal{K}, t>0$, one introduces the following operators on $\mathcal{H}_{\lambda}$

$$
a_{\lambda}^{\sharp}(t, g)=A_{\lambda}^{\sharp}\left(\frac{1}{\lambda} S\left(t / \lambda^{2}\right) g\right) .
$$

From the CCR one has

$$
\left[a_{\lambda}(t, g), a_{\lambda}^{\dagger}(s, f)\right]=\frac{1}{\lambda^{2}}\left\langle S\left(\frac{t-s}{\lambda^{2}}\right) g, f\right\rangle_{\mathcal{K}}
$$

which says roughly that these operators have auto-correlation time of the order $\lambda^{2}$. As $\lambda \rightarrow 0$, one expects these operators to become white noises, however, taking account of the previous section, the functional limit of (5.5) can be interpreted more concisely as

$$
\left[a(t, g), a^{\dagger}(s, f)\right]=\left\langle S_{+} g, f\right\rangle_{\mathcal{K}} \delta_{+}(t-s)+\left\langle S_{-} g, f\right\rangle_{\mathcal{K}} \delta_{-}(t-s)
$$

The limit operators $a^{\sharp}(t, g)$ are interpreted as the operators defined on $\mathcal{H}:=\Gamma_{+}\left(L^{2}(\mathbb{R}) \underline{\otimes} \mathcal{K}\right)$ by

$$
a(t, g) \Psi(\phi \underline{\otimes} f):=\left\langle\delta_{+}(t) \underline{\otimes} Z g+\delta_{-}(t) \underline{\otimes} Z^{\dagger} g, \phi \underline{\otimes} f\right\rangle \Psi(\phi \underline{\otimes} f)
$$

with $a^{\dagger}(t, g)$ the adjoint of $a(t, g)$.

THEOREM 5.1. The processes $\left\{a_{\lambda}^{\sharp}(t, g): t \geq 0, g \in \mathcal{K}\right\}$ on $\mathcal{H}_{\lambda}$ with the state $\Psi_{\lambda}$ converge in Fock vacuum expectation as $\lambda \rightarrow 0$ to the quantum white noises $\left\{a^{\sharp}(t, g): t \geq 0, g \in \mathcal{K}\right\}$ on $\mathcal{H}$ with the state $\Psi$. That is, for each integer $n \geq 0$, the following limits hold on $\mathcal{S}_{n}^{\prime}$,

$$
\lim _{\lambda \rightarrow 0^{+}}\left\langle\Psi_{\lambda}, a_{\lambda}^{\sharp(1)}\left(t_{1}, g_{1}\right) \cdots a_{\lambda}^{\sharp(n)}\left(t_{n}, g_{n}\right) \Psi_{\lambda}\right\rangle_{\mathcal{H}_{\lambda}}=\left\langle\Psi, a^{\sharp(1)}\left(t_{1}, g_{1}\right) \cdots a^{\sharp(n)}\left(t_{n}, g_{n}\right) \Psi\right\rangle_{\mathcal{H}},
$$

for all $t_{1}, \cdots, t_{n} \geq 0, g_{1}, \ldots, g_{n} \in \mathcal{K}$ and choices of creators and/or destroyers.

Proof. From the Gaussianity of the pre-limit processes it suffices to consider the two-point functions. For $\phi \in S_{2}$, let

$$
\begin{aligned}
I_{\lambda} & =\int_{\mathbb{R}^{+} \times \mathbb{R}^{+}} d s d t \phi(t, s) \frac{1}{\lambda^{2}}\left\langle S\left(\frac{t-s}{\lambda^{2}}\right) g, f\right\rangle_{\mathcal{K}} \\
& =\int_{0}^{\infty} d u \int_{-u / \lambda^{2}}^{u / \lambda^{2}} d \tau \phi\left(u+\lambda^{2} \tau, u-\lambda^{2} \tau\right)\langle S(\tau) g, f\rangle_{\mathcal{K}},
\end{aligned}
$$

where the change of variables $u=t+s, \tau=(t-s) / \lambda^{2}$ was made. If $J_{\theta}$ is the expression obtained from $I_{\lambda}$, by replacing the $t$-limits of integration by $\pm \infty$, then $\left|I_{\lambda}-J_{\lambda}\right| \rightarrow 0$ as $\lambda \rightarrow 0^{+}$uniformly since $f, g \in \mathcal{K}$. Moreover, since $\phi$ is $L^{2}$-regulated, $J_{\lambda}$ converges uniformly to

$$
\int_{0}^{\infty} d u\left\{\phi\left(u^{+}, u^{-}\right)\left\langle S_{+} g, f\right\rangle_{\mathcal{K}}+\phi\left(u^{-}, u^{+}\right)\left\langle S_{-} g, f\right\rangle_{\mathcal{K}}\right\}
$$


which establishes (5.6).

Next the CCR of the limit noises must be checked:

$$
\begin{aligned}
{\left[a(t, g), a^{\dagger}(s, f)\right]=} & \left\langle\delta_{+}(t) \underline{\otimes} Z g+\delta_{-}(t) \underline{\otimes} Z^{\dagger} g, \delta_{+}(s) \underline{\otimes} Z f+\delta_{-}(s) \underline{\otimes} Z^{\dagger} f\right\rangle_{\mathcal{H}} \\
= & \left\langle\left(Z^{\dagger}\right)^{2}+\frac{1}{2}\left(Z^{\dagger} Z+Z Z^{\dagger}\right) g, f\right\rangle_{\mathcal{K}} \delta_{+}(t-s) \\
& +\left\langle\left(Z^{2}+\frac{1}{2}\left(Z^{\dagger} Z+Z Z^{\dagger}\right) g, f\right\rangle_{\mathcal{K}} \delta_{-}(t-s)\right.
\end{aligned}
$$

but $\left(Z^{\dagger}\right)^{2}+\frac{1}{2}\left(Z^{\dagger} Z+Z Z^{\dagger}\right)=2 X^{2}-i(X Y+Y X)=\frac{1}{2} \Gamma+i \Sigma=S_{+}$and so (5.6) is recovered.

DEFINITION 5.2. Let $\Psi_{\lambda}(h)$ denote the exponential vector on $\mathcal{H}_{\lambda}=\Gamma_{+}(\mathcal{K})$ for $k \in \mathcal{K}$. Collective exponential vectors are defined, for $f \in \mathcal{S}$, by

$$
\Psi(f, k, \lambda):=\Psi_{\lambda}\left(\int_{0}^{\infty} d t f(t) S\left(t / \lambda^{2}\right) k\right) .
$$

The set of such collective exponential vectors is denoted $\operatorname{EX} P_{\lambda}(\mathcal{S}, \mathcal{K})$.

Noting that $\left\langle\Psi(f, k, \lambda), \Psi\left(f^{\prime}, k^{\prime}, \lambda\right)\right\rangle_{\mathcal{H}_{\lambda}} \rightarrow \exp \left\{\gamma\left(k, k^{\prime}\right)\left\langle f, f^{\prime}\right\rangle_{L^{2}\left(\mathbb{R}^{+}\right)}\right\}$as $\lambda \rightarrow 0^{+}$, it is natural to associate the limit $\Psi\left(f \underline{\otimes} \Gamma^{\frac{1}{2}} k\right)$ in $\mathcal{H}$ with $\Psi(f, k, \lambda)$ by virtue that $\gamma(k, k)=\langle\Gamma k, k\rangle_{\mathcal{K}}=\left\|\Gamma^{1 / 2} k\right\|_{\mathcal{K}^{2}}^{2}$.

In the following we shall understand all processes to be over a common domain $\mathcal{D}$ of $\mathcal{H}_{0}$, as outlined in Section 3 . A family of operators $\left(Z_{t}(\lambda)\right)$ on $\mathcal{H}_{0} \underline{\otimes} \mathcal{H}_{\lambda}$ is said to converge to a process $Z_{t}$ on $\mathcal{H}_{0} \underline{\otimes H}$ as $\lambda \rightarrow 0^{+}$weakly in matrix elements [9] if for all $n, t_{1}, \cdots, t_{n}$, and for all $u, u^{\prime} \in \mathcal{D}, f, f^{\prime} \in \mathcal{S}$ and $k, k^{\prime} \in \mathcal{K}$ one has

$$
\lim _{\lambda \rightarrow 0^{+}}\left\langle u \otimes \Psi(f, k, \lambda), Z_{t_{i}}(\lambda) \cdots Z_{t_{n}}(\lambda) u^{\prime} \otimes \Psi\left(f^{\prime}, k^{\prime}, \lambda\right)\right\rangle=\left\langle u \otimes \Psi(f \underline{\otimes} k), Z_{t_{i}}(\lambda) \cdots Z_{t_{n}}(\lambda) u^{\prime} \otimes \Psi\left(f^{\prime} \underline{\otimes} k^{\prime}\right)\right\rangle .
$$

Typically one would also like $Z_{t}(\lambda)$ to converge to an adapted process and for this reason the next definition is formulated. DEFINITION 5.3. A process $\left(Z_{t}(\lambda)\right)_{t \geq 0}$ on $\mathcal{H}_{0} \underline{\otimes \mathcal{H}}$ is said to be adaptable if, for each $t \geq 0$ and for all $s>t, g \in \mathcal{K}$, one has

$$
\left[Z_{t}(\lambda), a_{\lambda}^{\sharp}(s, g)\right]=O_{t, s}(\lambda),
$$

whereby a process $W=W_{t, s}(h)$ is said to be $O_{t, s}(\lambda)$ if $\int_{0}^{T} d s \int_{0}^{s} d t W_{t, s}(\lambda)$ vanishes in matrix element limits. Further one requires that, for all $u \in \mathcal{H}_{0}, f \in \mathcal{S}$ and $g \in \mathcal{K}$, and $\lambda$ in a neighborhood of $0^{+}$,

$$
\int_{0}^{t} d s\left\|Z_{s}(\lambda) u \otimes \Psi(f, k, \lambda)\right\|^{2}<C,
$$

where $C$ depends at most on $u, f$ and $g$.

Let $X_{t}^{\alpha \beta}(\lambda)$ be operators on $\mathcal{H}_{0} \underline{\otimes} \mathcal{H}$ which depend on $a_{\lambda}^{\sharp}(t, \cdot)$ for $s<t$. Note that, since the $a_{\lambda}^{\sharp}(s, g)$ have finite autocorrelation time, it does not follow that they will commute with $X_{t}^{\alpha \beta}(\lambda)$ for $s>t$, however the assumption shall be made that they are adaptable. Consider then ODES of the type

$$
\begin{aligned}
\frac{d}{d t} X_{t}(\lambda) & =a_{\lambda}^{\dagger}\left(t, g_{1}\right) X_{t}^{11}(\lambda) a_{\lambda}\left(t, g_{2}\right)+a_{\lambda}^{\dagger}\left(t, g_{3}\right) X_{t}^{10}(\lambda)+X_{t}^{01}(\lambda) a_{\lambda}\left(t, g_{4}\right)+X_{t}^{00}(\lambda) \\
X_{0}(\lambda) & =x_{0} \in \mathcal{B}\left(\mathcal{H}_{0}\right) .
\end{aligned}
$$

In this form the ODE has creators and destroyers in normal ordered from. The objective of the remainder of this section is to show that (5.12) converges to a well-defined QSDE under the ansatz that its coefficients are adaptable. For convenience, the K-state will be a fixed $g$ and it will be supposed that $\left\langle S_{ \pm} g, g\right\rangle_{\mathcal{K}}=\frac{1}{2}$ for the rest of this section. The $g$-dependence in the pre-limit and limit noises and collective exponential vectors will be dropped, and one notes that in this case the limit noise is just the fields $a_{*}(t)$ introduced in the previous section.

THEOREM 5.4. Suppose $X_{t}(\lambda)$ is the unique solution to a Wick ordered ODE with adaptable coeficients $X_{t}^{\alpha \beta}(\lambda)$ ) converging to processes $X_{t}^{\alpha \beta}$ so that the QSDE $d X_{t}=X_{t}^{\alpha \beta} \otimes d A_{t}^{\alpha \beta}, X_{0}=x_{0}$, has again unique solution $X_{t}$. Then $X_{t}(\lambda)$ converges to $X_{t}$, weakly in matrix elements as $\lambda \rightarrow 0^{+}$. 
Proof. Part (i). Note that one has now taken $\gamma(g, g)=1$ and writes $\Psi(f)$ for $\Psi\left(f \underline{\otimes} \Gamma^{\frac{1}{2}} g\right)$. Let $\varphi_{1}, \varphi_{2} \in \mathcal{H}_{0}$, $f, k \in L^{2}\left(\mathbb{R}^{+}\right)$, then as $\lambda \rightarrow 0$,

$$
\begin{aligned}
& \left\langle\varphi_{1} \underline{\otimes} \Psi(f, \lambda),\left\{X_{t}(\lambda)-x_{0}\right\} \varphi_{2} \underline{\otimes} \Psi(k, \lambda)\right\rangle \\
= & \int_{0}^{t} d s\left\langle\varphi_{1},\left\{\int_{0}^{\infty} d u \frac{f(u)^{*}}{\lambda^{2}}\left\langle g, S\left(\frac{u-s}{\lambda^{2}}\right) g\right\rangle X_{s}^{11}(\lambda) \int_{0}^{\infty} d v \frac{k(v)}{\lambda^{2}}\left\langle g, S\left(\frac{v-s}{\lambda^{2}}\right) g\right\rangle+\cdots\right\} \varphi_{2}\right\rangle_{\mathcal{H}_{0}} \\
\rightarrow & \int_{0}^{t} d s\left\langle\varphi_{1},\left\{f(s)^{*} X_{s}^{11} k(s)+f(s)^{*} X_{s}^{10}+X_{s}^{01} k(s)+X_{s}^{00}\right\} \varphi_{2}\right\rangle_{\mathcal{H}_{0}} \\
= & \left\langle\varphi_{1} \underline{\otimes} \Psi(f), \int_{0}^{t} X_{s}^{\alpha \beta} \otimes d A_{s}^{\alpha \beta} \varphi_{2} \underline{\otimes} \Psi(k)\right\rangle_{\mathcal{H}_{0} \otimes \mathcal{H}} .
\end{aligned}
$$

This amounts to saying that the approximation holds at the level of the QSDE, that is, $X_{t}(\lambda)$ converges to $X_{t}$ in first moment.

Part (ii). It is easy to see that higher moments $X_{t_{1}}(\lambda), \cdots, X_{t_{n}}(\lambda)$ decouple in collective coherent states, under the assumptions of the theorem, provided the $t_{j}$ are distinct. To deal with the case where several indices are equal, one actually shows that such moments converge to the appropriate equal time Ito products. This is established by first showing that if $Y_{t}(h)$ is a similarly described process, then the product $Y_{t}(h) X_{t}(h)$ converges to $X_{t} Y_{t}$ in QSDE.

Multiplying the pre-limit operators, one obtains

$$
\begin{aligned}
& \left(X_{t}(\lambda)-x_{0}\right)\left(Y_{t}(\lambda)-y_{0}\right) \\
= & \int_{0}^{t} d u \int_{0}^{t} d v\left\{a_{\lambda}^{\dagger}(u) X_{u}^{11}(\lambda) a_{\lambda}(u)+\cdots\right\}\left\{a_{\lambda}^{\dagger}(v) Y X_{v}^{11}(\lambda) a_{\lambda}(v)+\cdots\right\},
\end{aligned}
$$

there are $4 \times 4$ separate integrals here, examination of the first will be sufficient to determine the general pattern; one has

$$
\begin{aligned}
& \int_{0}^{t} d u \int_{0}^{t} d v a_{\lambda}^{\dagger}(u) X_{u}^{11}(\lambda) a_{\lambda}(u) a_{\lambda}^{\dagger}(v) Y_{v}^{11}(\lambda) a_{\lambda}(v) \\
= & \int_{0}^{t} d u \int_{0}^{t} d v a_{\lambda}^{\dagger}(u) X_{u}^{11}(\lambda)\left\{a_{\lambda}^{\dagger}(v) a_{\lambda}(u)+\frac{1}{\lambda^{2}}\left\langle g, S\left(\frac{u-v}{\lambda^{2}}\right) g\right\rangle\right\} Y_{v}^{11}(\lambda) a_{\lambda}(v) .
\end{aligned}
$$

If $v<u$, then $a_{\lambda}(u)$ can be commuted with $Y_{v}^{11}(\lambda)$ up to an $O_{u, v}(\lambda)$ error. Similarly, if $u<v$, then $a_{\lambda}^{\dagger}(v)$ can be commuted with $X_{u}^{11}(\lambda)$ up to an $O_{u, v}(\lambda)$ error, thus the above equals

$$
\begin{aligned}
& \int_{0}^{t} d u a_{\lambda}^{\dagger}(u) X_{u}^{11}(\lambda)\left\{\int_{0}^{u} d v a_{\lambda}^{\dagger}(v) Y_{v}^{11}(\lambda) a_{\lambda}(u)\right\} a_{\lambda}(v) \\
& +\int_{0}^{t} d u a_{\lambda}^{\dagger}(u) X_{u}^{11}(\lambda)\left\{\int_{0}^{u} d v O_{v, u}(\lambda) a_{\lambda}(u)\right\} a_{\lambda}(v) \\
& +\int_{0}^{t} d v a_{\lambda}^{\dagger}(v)\left\{\int_{0}^{v} d u a_{\lambda}^{\dagger}(u) X_{u}^{11}(\lambda) a_{\lambda}(u)\right\} Y_{v}^{11}(\lambda) a_{\lambda}(v) \\
& +\int_{0}^{t} d v \int_{0}^{v} d u a_{\lambda}^{\dagger}(v) a_{\lambda}(u) Y_{v}^{11}(\lambda) a_{\lambda}(u) O_{u, v}(\lambda) \\
& +\int_{0}^{t} d u \int_{0}^{t} d v \frac{1}{\lambda^{2}}\left\langle g, S\left(\frac{u-v}{\lambda^{2}}\right) g\right\rangle a_{\lambda}^{\dagger}(u) X_{u}^{11}(\lambda) Y_{v}^{11}(\lambda) a_{\lambda}(v) .
\end{aligned}
$$

In the limit $\lambda \rightarrow 0$ leads to

$$
\begin{aligned}
& \int_{0}^{t} X_{u}^{11}\left[\int_{0}^{u} Y_{v}^{11} \otimes d A_{v}^{11}\right] \otimes d A_{u}^{11} \\
& +\int_{0}^{t}\left[X_{u}^{11} d A_{u}^{11}\right] \int_{0}^{u} Y_{v}^{11} \otimes d A_{v}^{11}+\int_{0}^{t} X_{u}^{11} Y_{u}^{11} \otimes d A_{u}^{11} .
\end{aligned}
$$

The last term is an Ito correction. Such a term results whenever the pre-limit term $a_{\lambda}^{\dagger}(v) a_{\lambda}(u)$ is present and put into normal order. There are 4 such terms and they lead to the usual quantum Ito correction

$$
\lim _{\lambda \rightarrow 0^{+}}\left(X_{t}(\lambda)-x_{0}\right)\left(Y_{t}(\lambda)-y_{0}\right)=\left(X_{t}-x_{0}\right)\left(Y_{t}-y_{0}\right)
$$


Thus the Ito calculus is picked up in the limit and the convergence for all moments can be derived by induction.

Here we have not attempted a most general statement which might be formulated by defined processes as equivalence classes of sesquilinear forms on the appropriate space of exponential vectors. Instead, we assumed existence and uniqueness of solutions to begin with, however, situations where this can be established will be presented in the next section.

THEOREM 5.5. Let $X_{t}(\lambda)=x_{0}+\int_{0}^{t} d u\left\{a_{\lambda}^{\dagger}(u) X_{u}^{11}(\lambda) a_{\lambda}(u)+\cdots\right\}$ with adaptable coefficients $X_{t}^{\alpha \beta}(\lambda)$ such that $X_{t}(\lambda), X_{t}^{\alpha \beta}(\lambda)$ converge respectively in weak matrix elements to $X_{t}, X_{t}^{\alpha \beta}(\lambda)$ (uniformly for mixed matrix elements) us above, then the following limits hold us quantum Stratonovich integrals:

$$
\begin{aligned}
\int_{0}^{t} d s a_{\lambda}^{\dagger}(s) a_{\lambda}(s) X_{s}(\lambda) & \rightarrow \int_{0}^{t} d A_{s}^{11} \overline{X_{s}}, \\
\int_{0}^{t} d s X_{s}(\lambda) a_{\lambda}^{\dagger}(s) a_{\lambda}(s) & \rightarrow \int_{0}^{t} \overline{X_{s}} d A_{s}^{11}, \\
\int_{0}^{t} d s a_{\lambda}(s) X_{s}(\lambda) & \rightarrow \int_{0}^{t} d A_{s}^{01} \overline{X_{s}}, \\
\int_{0}^{t} d s X_{s}(\lambda) a_{\lambda}^{\dagger}(s) & \rightarrow \int_{0}^{t} \overline{X_{s}} d A_{s}^{10}, \\
\int_{0}^{t} d s X_{s}(\lambda) a_{\lambda}(s) & \rightarrow \int_{0}^{t} \overline{X_{s}} d A_{s}^{01}, \\
\int_{0}^{t} d s a_{\lambda}^{\dagger}(s) X_{s}(\lambda) & \rightarrow \int_{0}^{t} d A_{s}^{10} \overline{X_{s}} .
\end{aligned}
$$

Proof. The treatments of these limits are very similar, each involves at most one reordering, and it is enough to work through just one of them:

$$
\begin{aligned}
\int_{0}^{t} d s a_{\lambda}(s) X_{s}(\lambda)= & \int_{0}^{t} d s X_{s}(\lambda) a_{\lambda}(s) \\
& +\int_{0}^{t} d s \int_{0}^{s} d u\left[a_{\lambda}(s), a_{\lambda}^{\dagger}(u) X_{u}^{11}(\lambda) a_{\lambda}(u)+\cdots\right] \\
= & \int_{0}^{t} d s X_{s}(\lambda) a_{\lambda}(s)+\int_{0}^{t} d s \int_{0}^{s} d u \frac{1}{\lambda^{2}}\left\langle g, S\left(\frac{u-v}{\lambda^{2}}\right) g\right\rangle\left\{X_{u}^{11}(\lambda) a_{\lambda}(u)+X_{u}^{10}(\lambda)\right\} \\
& +\int_{0}^{t} d s \int_{0}^{s} d u a_{\lambda}^{\dagger}(u) O_{\lambda} a_{\lambda}(u) .
\end{aligned}
$$

Now, for $R_{\lambda}(u)$ the matrix elements of an adaptable process between arbitrary collective exponential states, one has

$$
\begin{aligned}
\lim _{\lambda \rightarrow 0^{+}} \int_{0}^{t} d s \int_{0}^{s} d u \frac{1}{\lambda^{2}}\left\langle g, S\left(\frac{u-v}{\lambda^{2}}\right) g\right\rangle R_{\lambda}(u) & =\lim _{\lambda \rightarrow 0^{+}} \int_{0}^{t} d s \int_{0}^{s / \lambda^{2}} d \tau\langle g, S(\tau) g\rangle R_{\lambda}\left(s-\tau / \lambda^{2}\right) \\
& =\frac{1}{2} \int_{0}^{t} R(s) d s,
\end{aligned}
$$

where $R(u)$ is the associated limit; from the standard notion of weak convergence in matrix elements from $\mathcal{H}_{0} \underline{\otimes} \mathcal{H}_{\lambda}$ to $\mathcal{H}_{0} \underline{\otimes \mathcal{H}}$ one sees that

$$
\begin{aligned}
\lim _{\lambda \rightarrow 0^{+}} \int_{0}^{t} d s a_{\lambda}(s) X_{s}(\lambda) & =\int_{0}^{t} X_{s} \otimes d A_{s}^{01}+\frac{1}{2} \int_{0}^{t}\left(X_{s}^{11} \otimes d A_{s}^{10}+X_{s}^{10} \otimes d A_{s}^{00}\right) \\
& =\int_{0}^{t} d A_{s}^{01} \overline{X_{s}} .
\end{aligned}
$$

\section{Asymptotic quantum stochastic limits. Uniformly convergent situa- tions}

The simplest way to bypass the adaptability ansatz introduced in the previous section is to consider only linear ODES. It is possible, however, to construct genuinely nonlinear examples starting from the linear case. This, in fact, is very 
natural: all important dynamical equations in science are nonlinear with the sole exception of the Schrödinger equation, this however leads to the Heisenberg evolution which is generally nonlinear. The program of this section is as follows. It is first of all shown that solutions $U_{t}(\lambda)$ to linear Wick-ordered ODES converge without adopting an adaptability ansatz. If the limit process $U_{t}$, is unitary, then it is shown that processes $X_{t}=U_{t}^{\dagger} x_{0} U_{t}$, for some $x_{0} \in \mathcal{B}\left(\mathcal{H}_{0}\right)$, satisfy nonlinear QSDEs. In a sense, this class of solutions is the most interesting as they provide examples of quantum dynamical variables having a symplectic evolution.

THEOREM 6.1. Let $x_{0}, C_{\alpha \beta} \in \mathcal{B}\left(\mathcal{H}_{0}\right)$ for $\alpha, \beta \in\{0,1\}$ and let $X_{t}(\lambda)$ be the solution to the linear ODE

$$
\dot{X}_{t}(\lambda)=a_{\lambda}^{\dagger}(t) C_{11} X_{t}(\lambda) a_{\lambda}(t)+a_{\lambda}^{\dagger}(t) C_{10} X_{t}(\lambda)+C_{01} X_{t}(\lambda) a_{\lambda}(t)+C_{00} X_{t}(\lambda)
$$

with $X_{0}(\lambda)=x_{0}$, then for all $u, v \in \mathcal{H}_{0}$ and $f, h \in \mathcal{S}$

$$
\lim _{\lambda \rightarrow 0^{+}}\left\langle u \underline{\otimes} \Psi(f, \lambda), X_{t}(\lambda) v_{2} \underline{\otimes} \Psi(k, \lambda)\right\rangle_{\mathcal{H}_{0} \underline{\otimes} \mathcal{H}}=\left\langle u \underline{\otimes} \Psi(f), X_{t} v \underline{\otimes} \Psi(k)\right\rangle_{\mathcal{H}_{0} \underline{\otimes} \mathcal{H}},
$$

where $X_{t}$ is the solution to the QSDE

$$
d X_{t}=\sum_{\alpha, \beta} C_{\alpha \beta} X_{t} \otimes d A_{t}^{\alpha \beta} ; \quad X_{0}=x_{0} .
$$

Proof. Let $a_{\lambda}^{\alpha}(t)=a_{\lambda}(t)$ for $\alpha=1$, and $=1$ for $\alpha=0$. Eq. (6.1) can be re-written as

$$
\dot{X}_{t}(\lambda)=\sum_{\alpha, \beta} a_{\lambda}^{\alpha}(t)^{\dagger} C_{\alpha \beta} X_{t}(\lambda) a_{\lambda}^{\beta}(t)
$$

As this is linear, the formal iterative series expansion exists,

$$
X_{t}(\lambda)=x_{0}+\sum_{n=1}^{\infty} X_{t}^{(n)}(\lambda)
$$

where

$$
\begin{aligned}
X_{t}^{(n)}(\lambda)= & \sum_{\alpha_{1}, \beta_{1}, \cdots, \alpha_{n}, \beta_{n}} \int_{t>t_{n}>\cdots>t_{1} \geq 0} d t_{1} \cdots d t_{n} \\
& \times\left(a_{\lambda}^{\alpha_{1}}\left(t_{1}\right) \cdots a_{\lambda}^{\alpha_{n}}\left(t_{n}\right)\right)^{\dagger} C_{\alpha_{1} \beta_{1}} \cdots C_{\alpha_{n} \beta_{n}} x_{0} a_{\lambda}^{\beta_{1}}\left(t_{1}\right) \cdots a_{\lambda}^{\beta_{n}}\left(t_{n}\right)
\end{aligned}
$$

In particular,

$$
\begin{aligned}
&\left\langle u \underline{\otimes} \Psi(f, \lambda), X_{t}(\lambda) v_{2} \underline{\otimes} \Psi(k, \lambda)\right\rangle_{\mathcal{H}_{0} \underline{\otimes H}}=\sum_{\begin{array}{c}
\alpha_{1}, \beta_{1}, \cdots, \alpha_{n}, \beta_{n} \\
\alpha_{1}
\end{array}} \int_{t>t_{n}>\cdots>t_{1} \geq 0} d t_{1} \cdots d t_{n}\left\langle u, C_{\alpha_{1} \beta_{1}} \cdots C_{\alpha_{n} \beta_{n}} x_{0} v\right\rangle_{\mathcal{H}_{0}} \\
& \times f_{\lambda}^{\alpha_{1}}\left(t_{1}\right)^{*} \cdots f_{\lambda}^{\alpha_{n}}\left(t_{n}\right)^{*} k_{\lambda}^{\beta_{1}}\left(t_{1}\right) \cdots k_{\lambda}^{\beta_{n}}\left(t_{n}\right)
\end{aligned}
$$

where $f_{\lambda}^{\alpha}(t):=\int_{0}^{\infty} f\left(t+\lambda^{2} u\right)\langle S(u) g, g\rangle d u$ for $\alpha=1$, and $=1$ for $\alpha=0$, etc.

Expression (6.3) is bounded by $c_{0}\|u\|\|v\|\left(c\langle\Gamma g, g\rangle\|f\|_{\infty}\|k\|_{\infty} t\right)^{n}$ where $c:=\max \left\|C_{\alpha \beta}\right\|$. The series expansion is therefore uniformly convergent, with (6.3) converging to

$$
\begin{aligned}
& \sum_{\alpha_{1}, \beta_{1}, \cdots, \alpha_{n}, \beta_{n}} \int_{t>t_{n}>\cdots>t_{1} \geq 0} d t_{1} \cdots d t_{n}\left\langle u, C_{\alpha_{1} \beta_{1}} \cdots C_{\alpha_{n} \beta_{n}} x_{0} v\right\rangle_{\mathcal{H}_{0}} \\
& \times f^{\alpha_{1}}\left(t_{1}\right)^{*} \cdots f^{\alpha_{n}}\left(t_{n}\right)^{*} k^{\beta_{1}}\left(t_{1}\right) \cdots k^{\beta_{n}}\left(t_{n}\right),
\end{aligned}
$$

where $f^{1}(t):=\left\langle S_{+} g, g\right\rangle f(t)^{*}$ and $f^{0}(t):=1$. Resumming gives the correct matrix element for the process $X_{t}$ described in the statement of the theorem.

The expediency of Theorem 6.1 compared to those employed in the papers of Accardi, Frigerio and Lu [9] comes about from the fact that here the limit is anticipated via white noise operators. The mechanism is transparent because the pre-limit and limit representations are Wick ordered. The difficulty encountered there was that the prelimit fields were Weyl ordered (which is generally the ordering natural to equations of elementary physics) and so enormous efforts were 
spent in re-ordering and the subsequent identification and treatment of negligible terms. Once it is known that the process converges, however, the adaptability ansatz can be dispensed with for the coefficients of the ODE. Part (ii) of Theorem 5.4 affirms that the multi-moment convergence of the process occurs. Therefore the following conclusion is reached.

THEOREM 6.2. Let $X_{t}(\lambda)$ be the solution of the linear ODE (6.1) then $X_{t}(\lambda)$ converges weakly to the solution of the QSDE (6.2) in matrix elements.

The problem of obtaining a unitary process $U_{t}$ obeying a linear QSDE has been tackled $[12,171$ and can be summarized in the next theorem. Here $a^{\sharp}(t)=a^{\sharp}(t, g)$ with $\left\langle S_{+} g, g\right\rangle$ taken as $\kappa=\frac{1}{2} \gamma+i \sigma$. The CCR for the noise is then $\left[a(t), a^{\dagger}(s)\right]=\kappa \delta_{+}(t-s)+\kappa_{*} \delta_{-}(t-s)$. With the identifications $d A_{t}^{11}=a^{\dagger}(t) a(t) d t, d A_{t}^{10}=a^{\dagger}(t) d t, d A_{t}^{01}=a(t) d t$ and $d A_{t}^{00}=d t$ one is led to the nontrivial component of the lto table being $d A_{1}^{\alpha 1} d A_{t}^{1 \beta}=\gamma d A_{t}^{\alpha \beta}$.

THEOREM 6.3. The general unitary process $[23]\left(U_{t}\right)_{t \geq 0}$ driven by white noise processes $a^{\sharp}(t)$ is given by $\left(U_{0}=1\right)$

$$
\begin{aligned}
\dot{U}_{t} & =\left[1, \frac{1}{\sqrt{\gamma}} a^{\dagger}(t)\right]\left[\begin{array}{cc}
-i H-\frac{1}{2} L^{\dagger} L & -L^{\dagger} W \\
L & W-1
\end{array}\right] U_{t}\left[\begin{array}{c}
1 \\
\frac{1}{\sqrt{\gamma}} a(t)
\end{array}\right] \\
& =-i\left[E a^{\dagger}(t) a(t)+F a^{\dagger}(t)+F^{\dagger} a(t)+G\right]
\end{aligned}
$$

where $E, H, G$ are self-adjoint and $W$ is unitary on $\mathcal{H}_{0}$, and the coeficients of the Ito QSDE (6.4) are related to those of the Stratonovich QSDE (6.5) by the relations

$$
W=\frac{1-i \kappa^{*} E}{1+i \kappa E}, \quad L=-i \sqrt{\gamma}(1+i \kappa E)^{-1} F, \quad H=G+F^{\dagger} \frac{\sigma-|\kappa|^{2} E}{1+|\kappa|^{2} E} F
$$

One notes that the Stratonovich QSDE takes the form $\dot{U}_{t}=-i \Upsilon_{t} U_{t}$, where $\Upsilon_{t}=E a^{\dagger}(t) a(t)+F a^{\dagger}(t)+F^{\dagger} a(t)+G$ can be interpreted as a quantum stochastic Hamiltonian.

THEOREM 6.4. Let $E, F, G \in \mathcal{B}\left(\mathcal{H}_{0}\right)$ with $E$ and $G$ self-adjoint then the family of processes $U_{\lambda}(t)$ satisfying the ODES

$$
\dot{U}_{t}(\lambda)=-i \Upsilon_{t}(\lambda) U_{t}(\lambda), \quad U_{0}(\lambda)=1
$$

with

$$
\Upsilon_{t}(\lambda)=E a_{t}^{\dagger}(\lambda) a_{t}(\lambda)+F a_{t}^{\dagger}(\lambda)+F^{\dagger} a_{t}(\lambda)+G,
$$

converges weakly in matrix elements to the unitary quantum stochastic process described in Theorem 6.3.

Proof. One begins by noting that

$$
\begin{aligned}
{\left[a_{\lambda}(t), U_{t}(\lambda)\right] } & =-i\left[a_{\lambda}(t), \int_{0}^{t} d s \Upsilon_{s}(\lambda) U_{s}(\lambda)\right] \\
& =-i \int_{0}^{t} d s \frac{1}{\lambda^{2}}\left\langle g, S\left(\frac{t-s}{\lambda^{2}}\right) g\right\rangle\left\{E a_{s}(\lambda)+F\right\} U_{s}(\lambda) \\
& \equiv-i\left\langle S_{+} g, g\right\rangle\left\{E a_{t}(\lambda)+F\right\} U_{t}(\lambda)+O(\lambda) .
\end{aligned}
$$

Rearranging the ODE from Weyl to Wick ordered form gives

$$
\begin{aligned}
\dot{U}_{t}(\lambda)= & \frac{1}{\gamma} a_{\lambda}^{\dagger}(t)(W-1) U_{t} a_{\lambda}(t)+\frac{1}{\sqrt{\gamma}} a_{\lambda}^{\dagger}(t) L U_{t} \\
& -\frac{1}{\sqrt{\gamma}} L^{\dagger} W U_{t} a_{\lambda}(t)-\left(\frac{1}{2} L^{\dagger} L+i H\right) U_{t}+O(\lambda) ;
\end{aligned}
$$

one recognizes the pre-limit form of (6.4) and the results of the previous section imply convergence.

The weak convergence in matrix elements, however, gives much more.

THEOREM 6.5. Let $x_{o} \in \mathcal{B}\left(\mathcal{H}_{0}\right)$, and set $X_{t}(\lambda)=U_{t}^{\dagger}(\lambda) x_{0} U_{t}(\lambda)$, then $X_{t}(\lambda)$ converges weakly in matrix elements to the quantum stochastic process $X_{t}=U_{t}^{\dagger} x_{0} U_{t}$. 
Given $X_{t}=U_{t}^{\dagger} x_{0} U_{t}$, then one has the stochastic evolution equations

$$
\dot{X}_{t}=\dot{U}_{t}^{\dagger} x_{0} U_{t}+U_{t}^{\dagger} x_{0} \dot{U}_{t}=\frac{1}{i}\left[X_{t}, \Theta_{t}\right]
$$

where $\Theta_{t}:=U_{t}^{\dagger} \Upsilon U_{t}$. The second part takes the form of a stochastic Heisenberg equation. It is a straightforward calculation to show, either by converting to the Hudson-Parthasarathy calculus using the quantum Ito formula and inverting back or by the now standard manipulations using the white noise calculus to put to Wick order, that the QSDE for $X_{t}$, is

$$
\begin{aligned}
\dot{X}_{t}= & \frac{1}{\gamma} a^{\dagger}(t)\left(W_{t}^{\dagger} X_{t} W_{t}-X_{t}\right) a(t) \\
& +\frac{1}{\sqrt{\gamma}} a^{\dagger}(t) W_{t}^{\dagger}\left[X_{t}, L_{t}\right]-\frac{1}{\sqrt{\gamma}}\left[X_{t}, L_{t}^{\dagger}\right] W_{t} U_{t} a_{\lambda}(t) \\
& -\frac{1}{2}\left[L_{t}^{\dagger}, X_{t}\right] L_{t}-\frac{1}{2} L_{t}^{\dagger}\left[X_{t}, L_{t}\right]-i\left[X_{t}, H_{t}\right] .
\end{aligned}
$$

The Stratonovich version of this will now be computed.

LEMMA 6.6. Let $U_{t}$ be the solution to the QSDEs (6.4) and (6.5), then one can perform the following commutations under the integral:

$$
\begin{aligned}
U_{t}^{\dagger} a^{\dagger}(t)= & \left\{a^{\dagger}(t) U_{t}^{\dagger}+i \kappa^{*} U_{t}^{\dagger} F^{\dagger}\right\}\left(1-i \kappa^{*} E\right)^{-1}, \\
U_{t}^{\dagger} a(t)= & \left\{a(t) U_{t}^{\dagger}-i \kappa U_{t}^{\dagger} F\right\}(1+i \kappa E)^{-1}, \\
U_{t}^{\dagger} a^{\dagger}(t) a(t)= & \left\{a^{\dagger}(t) a(t) U_{t}^{\dagger}-i \kappa a^{\dagger}(t) U_{t}^{\dagger}\left(1-i \kappa^{*} E\right)^{-1} F\right. \\
& +i \kappa^{*} a(t) U_{t}^{\dagger}(1+i \kappa E)^{-1} F^{\dagger}+|\kappa|^{2} U_{t}^{\dagger} F\left(1-i \kappa^{*} E\right)^{-1} F^{\dagger} \\
& \left.+|\kappa|^{2} U_{t}^{\dagger} F^{\dagger}\left(1-i \kappa^{*} E\right)^{-1} F\right\}(1-2 \sigma E)^{-1} .
\end{aligned}
$$

Proof. Before demonstrating the proof it is important to point out that (6.12) cannot be obtained from (6.10) and (6.11); this is due to the afore-mentioned nonassociativity (that is, the product of Stratonovich integrands is not defined as the Stratonovich integrand of the products). To be consistent, the left-hand side of (6.12) should be written as U/at(t)a(t); the symbol U/at (t)Z(t) has not been defined. However, the underbar and overbar notation has been dropped for convenience. To prove (6.10) one has directly

$$
\begin{aligned}
{\left[a(t), U_{t}\right] } & =-i \int_{0}^{t}\left[a(t),\left(E a^{\dagger}(t) a(t)+F a^{\dagger}(t)+F^{\dagger} a(t)+G\right) U_{s}\right] d s \\
& =-i \int_{0}^{t}(E a(s)+F)\left\{\kappa \delta_{+}(t-s)+\kappa_{*} \delta_{-}(t-s)\right\} U_{s} \\
& =-i \kappa(E a(s)+F) U_{t} .
\end{aligned}
$$

The right-hand side is not properly normal ordered; however, it can be rearranged to give

$$
a(t) U_{t}=(1+i \kappa E)^{-1}\left\{U_{t} a(t)-i \kappa F U_{t}\right\} .
$$

Note that this can be deduced from the pre-limit expressions and corresponds then to (6.7). Eq. (6.10) is the conjugate equation to (6.13). The derivation of (6.11) is similar.

To derive (6.12), note first of all that

$$
\begin{aligned}
{\left[a^{\dagger}(t) a(t), \Upsilon_{t}\right]=} & E a^{\dagger}(t) a(s)\left\{\kappa \delta_{+}(t-s)+\kappa_{*} \delta_{-}(t-s)\right\}-E a^{\dagger}(s) a(t)\left\{\kappa \delta_{+}(s-t)+\kappa_{*} \delta_{-}(s-t)\right\} \\
& +F a^{\dagger}(t)\left\{\kappa \delta_{+}(t-s)+\kappa_{*} \delta_{-}(t-s)\right\}-F^{\dagger} a(t)\left\{\kappa \delta_{+}(s-t)+\kappa_{*} \delta_{-}(s-t)\right\} .
\end{aligned}
$$

Therefore,

$$
\begin{aligned}
{\left[a^{\dagger}(t) a(t), U_{t}\right] } & =-i-i \int_{0}^{t}\left[a^{\dagger}(t) a(t), \Upsilon_{s} U_{s}\right] d s \\
& =-i E a^{\dagger}(t) a(t)\left(\kappa-\kappa^{*}\right) U_{t}-i \kappa F a^{\dagger}(t) U_{t}+i \kappa^{*} F^{\dagger} a(t) U_{t}
\end{aligned}
$$


Again (6.15) is not wholly ordered, however, whereas the manipulations are not associative, they are linear and so $a^{\sharp}(t) U_{t}$ can be replaced using (6.13), etc. This will lead to the conjugate equation to (6.12).

The first form of the Heisenberg equation (6.8) can be written as

$$
\dot{X}_{t}=U_{t}^{\dagger}\left\{a^{\dagger}(t) a(t) \frac{1}{i}\left[x_{0}, E\right]+a^{\dagger}(t) \frac{1}{i}\left[x_{0}, F\right]+\frac{1}{i}\left[x_{0}, F^{\dagger}\right] a(t)+\frac{1}{i}\left[x_{0}, G\right]\right\} U_{t} .
$$

The conversion to Stratonovich form (left-handed) can now be made using the lemma. One obtains

$$
\begin{aligned}
\dot{X}_{t}= & a^{\dagger}(t) a(t)\left(1-2 \sigma E_{t}\right)^{-1} \frac{1}{i}\left[X_{t}, E_{t}\right] \\
& +a^{\dagger}(t)\left\{-i \kappa\left(1-i \kappa^{*} E_{t}\right)^{-1} F_{t}\left(1-\sigma E_{t}\right)^{-1} \frac{1}{i}\left[X_{t}, E_{t}\right]+\left(1-i \kappa^{*} E_{t}\right)^{-1} \frac{1}{i}\left[X_{t}, F_{t}\right]\right\} \\
& +a(t)\left\{i \kappa^{*}\left(1+i \kappa E_{t}\right)^{-1} F_{t}^{\dagger}\left(1-\sigma E_{t}\right)^{-1} \frac{1}{i}\left[X_{t}, E_{t}\right]+\left(1+i \kappa E_{t}\right)^{-1} \frac{1}{i}\left[X_{t}, F_{t}^{\dagger}\right]\right\} \\
& +\left\{\frac{1}{i}\left[X_{t}, H_{t}\right]+|\kappa|^{2} F_{t}\left(1+i \kappa E_{t}\right)^{-1} F_{t}^{\dagger}\left(1-2 \sigma E_{t}\right)^{-1} \frac{1}{i}\left[X_{t}, E_{t}\right]\right. \\
& +|\kappa|^{2} F_{t}^{\dagger}\left(1-i \kappa^{*} E_{t}\right)^{-1} F_{t}\left(1-2 \sigma E_{t}\right)^{-1} \frac{1}{i}\left[X_{t}, E_{t}\right] \\
& +i \kappa^{*} F_{t}^{\dagger}\left(1-i \kappa^{*} E_{t}\right)^{-1} \frac{1}{i}\left[X_{t}, F_{t}\right]-\kappa F_{t}\left(1+i \kappa E_{t}\right)^{-1} \frac{1}{i}\left[X_{t}, F_{t}^{\dagger}\right] .
\end{aligned}
$$

Eqs. (6.9) and (6.17) are equivalent and give the most general Heisenberg equation for this situation.

\section{Remarks}

The dynamical evolutions considered in this section are broad enough to include the classical SDEs considered in Sections 2 and 3.

Let $x_{0}, p_{0}$ be canonically conjugate variables on $\mathcal{H}_{0}$. Here the specification $\kappa=\frac{1}{2}, W=1, L=-i \tilde{\sigma}\left(x_{0}\right) p_{0}$, and $G=\frac{1}{2}\left(\tilde{v}\left(x_{0}\right) p_{0}+p \tilde{v}\left(x_{0}\right)\right)$ leads to (6.9) being the diffusion described in (4.8b). The QSDE has unbounded operator coefficients, but this can be dealt with using standard techniques [24]. Here $E=0, F=p_{0} \tilde{\sigma}\left(x_{0}\right)$, and $H=G$, and so (6.17) reduces to

Here $P_{t}=U_{t}^{\dagger} p_{0} U_{t}$ and since the evolution is unitary, $\left[X_{t}, P_{t}\right]=i$ for each $t \geq 0$. The last term in (6.18) is therefore $\frac{1}{2} i\left[\tilde{\sigma}\left(X_{t}\right), P_{t}\right] \tilde{\sigma}\left(X_{t}\right)=-\frac{i}{2} \tilde{\sigma}^{\prime}\left(X_{t}\right) \tilde{\sigma}\left(X_{t}\right)$ which is the correct drift term, and so (6.17) reduces to (4.8a). The classical relations are therefore recovered.

Likewise, to obtain Eqs. (4.9) for the Poisson driven processes, one sets $\kappa=\frac{1}{2}$ and takes $E=F=F^{\dagger}=H-$ $\frac{1}{2}\left(p_{0} v\left(x_{0}\right)+v\left(x_{0}\right) p_{0}\right)=\frac{1}{2}\left(p_{0} \mu\left(x_{0}\right)+\mu\left(x_{0}\right) p_{0}\right)$. In this case $W$ can be represented as the change-of-variable operator

$(W f)(x)==\sqrt{\frac{d u}{d x}} f(u(x))$ where $u=u(x):=x+\tilde{\mu}(x)$. In this case $W^{\dagger} x_{0} W=x_{0}+\tilde{\mu}\left(x_{0}\right)$, with $x_{0}$ interpreted as the multiplication by $x$ operator on $\mathcal{H}_{0}:=L^{2}(\mathbb{R})$.

Acknowledgment

This work was supported by the Irish Higher Education Authority EU Presidency Research Fellowship Program and the author would like to thank Professor D. Heffernan (Maynooth) for many stimulating discussions while writing this paper. The author also acknowledges many valuable comments made by the referee which have lead to an improvement to the original version.

\section{REFERENCES}

[1] A. D. Viencel and M. I. Freidlin: Fluctuations In Dynamical Systems Subjected to Small Random Perturbations, Nauka, Moscow 1979 (in Russian)

[2] K. Sobczyk: Stochastic Differential Equations, Mathematics in Applications, Vol. 40, Kluwer, Dordrecht 1991.

[3] R. Z. Khasminskii: A limit theorem for the solution of a differential equation with random right hand side, Teoria Vieroyatn. Prim. 11, No. 2 (1966).

[4] E. Wong and M. Zakai: ht. J. Eng. Sci. 3 (1965), 213-229.

[5] R. L. Stratonovich: SIAM J. Control 4 (1966), 362-371.

[6] L. van Hove: Physica 21 (1955), 617-640.

[7] W. H. Louisell: Quantum Statistical Properties of Radiation, Wiley Classics Library Edition, New York 1990. 
[8] L. Accardi, J. Cough and Y. G. Lu: Rep. Math. Phys. 36 (2/3) (1995), 155-187.

[9] L. Accardi, A. Frigerio and Y. G. Lu: Commun. Math. Phys. 131 (1990) 537-570.

[10 J. Gough, C. R. Acad, Sci. Paris 326, Serie I (1998), 981-985.

[11] C. W. Gardiner and M. J. Collet: Phys. Rev. A 31 (1985), 3761-3774.

[12] R. L. Hudson and K. R. Parthasarathy: Commun. Math. Phys. 93 (1984), 301-323.

131 R. L. Hudson and R. Streater: Phys. Left. A 277 (1981).

[14] A. M. Chebotarev: Mat. Zametki 60, No. 5 (1996), 726-750.

[15] P. Talkner: Ann. Phys. 167 (1980), 390.

[16] J. Gough: Causal structure of quantum stochastic integrators, Theoretical Mathematical Physics 111, No. 2, May (1997), 218-233 (with English translation 563-575).

[17] J. Gough: Non-commutative Ito and Stratonovich noise and stochastic evolutions, Theoretical and Mathematical Physics 113, No. 2, November (1997), 276-284 (with English translation 1431-1437).

[18] J. Gough: The Stratonovich interpretation of quantum stochastic approximations, Journ. Potential Analysis, Volume 11, Issue 3, pp 213-233, November 1999

[19] I. I. Gihman and A. V. Skorohod: Stochastic Differential Equations, Springer, Berlin 1974.

[20] K. R. Parthasarathy: An Introduction to Quantum Stochastic Calculus, Monographs in Mathematics, Birkhauser, Base1 1992.

[21] J. Dieudonne: Foundations of Modern Analysis, Academic Press, New York 1968.

[22] F. A. Berezin: Vsp. Fiz. Nauk. 132 (November 1980), 497-548.

[23] The Ito QSDEs with $W=-1$ are not convertible to standard $(\kappa=1)$ Stratonovich form as the first of Eq. (6.6) is noninvertible. The reflection process is an example.

[24] K. B. Sinha: Quantum Stochastic Calculus and Applications-A Review, Probability Towards 2000, Springer, New York 1998. 